JOURNAL OF

SYMPLECTIC GEOMETRY

Volume 8, Number 2, 143-187, 2010

\title{
ALMOST TORIC SYMPLECTIC FOUR-MANIFOLDS
}

\author{
Naichung Conan Leung and Margaret Symington
}

\begin{abstract}
Almost toric manifolds form a class of singular Lagrangian fibered symplectic manifolds that include both toric manifolds and the K3 surface. We classify closed almost toric four-manifolds up to diffeomorphism and indicate precisely the structure of all almost toric fibrations of closed symplectic four-manifolds. A key step in the proof is a geometric classification of the singular integral affine structures that can occur on the base of an almost toric fibration of a closed four-manifold. As a byproduct we provide a geometric explanation for why a generic Lagrangian fibration over the two-sphere must have 24 singular fibers.
\end{abstract}

\section{Introduction}

Almost toric manifolds, introduced by the second author in [39], are symplectic manifolds equipped with a fibration structure that generalizes toric manifolds while retaining some of their geometric features and rigidity. Accordingly, almost toric manifolds lie at the interface of symplectic topology, toric geometry, integrable systems and, in dimension four, mirror symmetry. They enjoy the property (similar to toric manifolds) that much symplectic and topological information is encoded in the base of the fibration; they, like toric fibrations, can be used to efficiently describe certain symplectic surgeries such as symplectic sums $[\mathbf{2 8}, \mathbf{3 6}]$ and rational blowdowns $[\mathbf{3 7}, \mathbf{3 8}]$; they accommodate singularities that are typical in an integrable system (focus-focus and elliptic singularities) $[\mathbf{3}, \mathbf{1 0}, \mathbf{3 3}]$; and furthermore, generic special Lagrangian fibrations of K3 surfaces - of interest in mirror symmetry (cf. $[\mathbf{1 8}, \mathbf{2 0}, \mathbf{3 1}, \mathbf{3 5}]$ ) — are almost toric fibrations.

The main result of this paper is summarized in Table 1, which includes a list of all closed four-manifolds that admit an almost toric fibration (the content of Theorem 2.1). An informal definition of a generic almost toric fibration in dimension four (made precise in Definition 2.2) is a surjective map $\pi:(M, \omega) \rightarrow B$ that gives the symplectic four-manifold $(M, \omega)$ the structure 
Table 1. Closed almost toric four-manifolds

\begin{tabular}{|c|c|c|c|}
\hline Base & \# Of nodes & \# Of vertices & Total space \\
\hline$D^{2}$ & $n \geq 0$ & $k \geq \max (0,3-n)$ & $\begin{array}{c}\mathbb{C} P^{2} \#(n+k-3) \overline{\mathbb{C} P}^{2} \text { or } \\
S^{2} \times S^{2}(\text { if } n+k=4)\end{array}$ \\
\hline$S^{1} \times I$ & $n \geq 0$ & 0 & $\begin{array}{c}S^{2} \times T^{2} \text { or } \\
\left(S^{2} \tilde{\times} T^{2}\right) \# n \overline{\mathbb{C P}}^{2}\end{array}$ \\
\hline Möbius band & $n \geq 0$ & 0 & $\begin{array}{c}S^{2} \times T^{2} \text { or } \\
\left(S^{2} \tilde{\times} T^{2}\right) \# n \overline{\mathbb{C} P}^{2}\end{array}$ \\
\hline$S^{2}$ & 24 & 0 & K3 surface \\
\hline $\mathbb{R} P^{2}$ & 12 & 0 & Enriques surface \\
\hline$T^{2}$ & 0 & 0 & $\begin{array}{c}T^{2} \text { bundles } \\
M\left\{\left(\begin{array}{cc}1 & 0 \\
0 & 1\end{array}\right),\left(\begin{array}{ll}1 & k \\
0 & 1\end{array}\right) ; m, n\right\}, \\
k, m, n \in \mathbb{Z}\end{array}$ \\
\hline Klein bottle & 0 & 0 & $\begin{array}{c}T^{2} \text { bundles } \\
N\left\{\left(\begin{array}{cc}1 & \delta \\
0 & -1\end{array}\right),\left(\begin{array}{ll}1 & k \\
0 & 1\end{array}\right) ; a, \epsilon\right\} \\
\delta, \epsilon \in\left\{\begin{array}{l}0,1\} \\
0,1\end{array}\right\} \text { and } k, a \in \mathbb{Z}\end{array}$ \\
\hline
\end{tabular}

$S^{2} \tilde{\times} T^{2}$ denotes the non-trivial sphere bundle over the torus.

In the expressions $M(A, B ; m, n)$ and $N(A, B ; a, \epsilon)$, the matrices $A$ and $B$ are the monodromy matrices along generators of the fundamental group (where $A B=B^{-1} A$ when the base is a Klein bottle) and $(m, n)$ and $(a, \epsilon)$ are the Euler classes of the bundles (see Section 6.4).

of a Lagrangian fibration in which each fiber has a neighborhood that is fiber preserving diffeomorphic to a neighborhood of a fiber in a toric manifold or a genus one Lefschetz fibration.

Because the fibration is Lagrangian, the base $B$ inherits an integral affine structure (Definition 2.4) with isolated singularities on the interior of $B$, namely nodes, which are the images of isolated two-dimensional singular fibers. As for the moment map image of a toric manifold, the boundary of $B$ is piecewise linear with respect to the integral affine structure. The restrictions on the base $B$ imposed by its integral affine geometry yield Theorem 5.2, which classifies the integral affine bases. Theorems 5.2 and 6.1, which implies that the only almost toric manifolds that fiber over the disk are rational surfaces, together account for the fact that the list of almost toric manifolds in Table 1 is complete. The proof of Theorem 5.2 includes a proof that an integral affine structure on $S^{2}$ must have 24 nodes, a point 
of interest in mirror symmetry as it implies a generic Lagrangian fibration over $S^{2}$ must have 24 singular fibers. ${ }^{1}$

Except for the Enriques surface, it is not a surprise that each of the total spaces in Table 1 admits an almost toric fibration. The presence of the Enriques surface is interesting because the fibration is over $\mathbb{R} P^{2}$, rather than over $\mathbb{C} P^{1}$ as it is for a holomorphically fibered Enriques surface. The contribution of this paper concerning fibrations over the Klein bottle is the determination of which torus bundles over the Klein bottle admit a symplectic structure with respect to which the fibration is Lagrangian. The existence of almost toric fibrations on the other manifolds listed in Table 1 is drawn from known results, as detailed in Section 6.

The paper is structured as follows. After motivating and defining the necessary terms and stating the main results in Section 2, we explain in Section 3 how the total space of a toric or almost toric fibration is determined by the base equipped with its integral affine structure. In Section 4 we describe two surgeries, one that changes the fibration but not the manifold, and another that shows how blowing up can introduce a node in the base of the fibration. Section 5 is devoted to the classification of integral affine manifolds that can appear as the base of a closed almost toric manifold. This classification is critical to showing the completeness of the list of manifolds in Table 1. To prove that the base of an almost toric fibration must have non-negative Euler characteristic, we make precise the heuristic that nodes contribute non-negative curvature to the base. (Note that the non-trivial monodromy around a node precludes the existence of a metric compatible with the integral affine structure.) A key lemma states that any collection of 12 nodes around which the monodromy is trivial contributes $2 \pi$ to the total curvature of the base (Lemma 5.4), thereby explaining the need for 24 nodes in an integral affine structure on the two-sphere. We also prove Theorem 6.1, which asserts that rational surfaces are the only closed manifolds that admit an almost toric fibration over the disk. In Section 7 we establish the existence and completeness of our list of the manifolds fibering over each base and address any diffeomorphism equivalences. In the last section we comment on other classifications one might try to make.

\section{Background and results}

A symplectic manifold of dimension $2 n$ is toric if it is equipped with an effective Hamiltonian $T^{n}$ action. Toric manifolds are well studied for their beautiful geometric properties (e.g., $[\mathbf{2}, \mathbf{1 2}]$ ) and their relevance to mirror symmetry (cf. [4]). Delzant's Theorem [8] asserts a fundamental property of closed toric manifolds: the manifold, symplectic structure and torus action

\footnotetext{
${ }^{1}$ This fact was also proved by Kontsevich and Soibelman $[\mathbf{2 4}]$.
} 
are completely determined by a polytope in $\mathbb{R}^{n}$, the image of the moment $\operatorname{map} \mu:(M, \omega) \rightarrow \mathbb{R}^{n}$.

A toric manifold is an example of a (completely) integrable system, namely a symplectic $2 n$-manifold $(M, \omega)$ equipped with a collection of $n$ functionally independent Poisson commuting functions $F_{i}:(M, \omega) \rightarrow \mathbb{R}$. The components of the moment map Poisson commute because the induced Hamiltonian vector fields commute. Consequently, the map $F:=\left(F_{1}, \ldots, F_{n}\right)$ induces a foliation of the total space such that $\omega$ vanishes along leaves, which are thereby Lagrangian.

In keeping with holomorphic fibrations, whose fibers can have varying topology, when $F$ is a proper map we regard the induced foliation as a fibration. Accordingly, we make the following definitions.

Definition 2.1. Consider a symplectic $2 n$-manifold $(M, \omega)$ and a proper surjective map $\pi:(M, \omega) \rightarrow B$ to a topological space of half the dimension. Assume the preimages of $\pi$ are connected. If $B$ can be covered by open sets $U_{\alpha}$ such that for each $\alpha$ there is a map $\phi_{\alpha}: U_{\alpha} \rightarrow \mathbb{R}^{n}$ such that $\phi_{\alpha} \circ$ $\left.\pi\right|_{\pi^{-1}\left(U_{\alpha}\right)}$ defines a completely integrable system $F_{\alpha}: \pi^{-1}\left(U_{\alpha}\right) \rightarrow \mathbb{R}^{n}$, then $\pi:(M, \omega) \rightarrow B$ is a Lagrangian fibration. If $\pi$ defines a locally trivial fibration over $B$ then we call $\pi:(M, \omega) \rightarrow B$ a regular Lagrangian fibration.

Throughout this paper we use $B_{0}$ to denote the set of regular values of $\pi$, i.e., the portion of $B$ over which $\pi$ defines a regular Lagrangian fibration. Note that because the fibers of $\pi$ are compact, the Arnold-Liouville theorem (cf. [1]) implies that the regular fibers are tori.

The moment map of a toric manifold is a Lagrangian fibration whose singular fibers are points and circles. To generalize to almost toric manifolds we allow one other type of singularity - the isolated singularity that appears in Lefschetz fibrations. Recall that a Lefschetz fibration is a map from an oriented four-manifold to the oriented two-sphere such that the map is a submersion except at a finite set of points where the singularity is modeled by $\left(z_{1}, z_{2}\right) \rightarrow z_{1}^{2}+z_{2}^{2}$. The importance of Lefschetz fibrations in symplectic topology emerged after Donaldson [9] showed that, after blowing up a sufficient number of points, any closed symplectic four-manifold admits a Lefschetz fibration and Gompf [14] showed how to put a symplectic structure on any four-manifold equipped with a Lefschetz fibration. In those constructions the fibration is symplectic whereas in our setting it is Lagrangian, thereby forcing the fibers to have genus one. An important example of a Lagrangian Lefschetz fibration is a generic elliptic fibration of a K3 surface; while the fibration is holomorphic with respect to the Kähler form on the K3, the symplectic form can be modified (by performing a hyperkäher rotation) so that the fibers of the given fibration become Lagrangian. (See Section 6.3.) We refer to the singular fibers of a genus one Lefschetz fibration as nodal fibers in accordance with the terminology 
of elliptic fibrations. Meanwhile, the singularity in a Lagrangian Lefschetz fibration is known as a focus-focus singularity in the integrable systems literature.

We now give the precise definition of an almost toric manifold:

Definition 2.2. An almost toric fibration of a symplectic four-manifold $(M, \omega)$ is a Lagrangian fibration $\pi:(M, \omega) \rightarrow B$ such that any point of $(M, \omega)$ has a Darboux neighborhood (with symplectic form $d x_{1} \wedge d y_{1}+$ $\left.d x_{2} \wedge d y_{2}\right)$ in which the map $\pi$ has one of the following forms:

$$
\begin{aligned}
\pi(x, y) & =\left(x_{1}, x_{2}\right), \quad \text { regular point, } \\
\pi(x, y) & =\left(x_{1}, x_{2}^{2}+y_{2}^{2}\right), \quad \text { elliptic, corank one, } \\
\pi(x, y) & =\left(x_{1}^{2}+y_{1}^{2}, x_{2}^{2}+y_{2}^{2}\right), \quad \text { elliptic, corank two or } \\
\left(\pi_{1}, \pi_{2}\right)(x, y) & =\left(x_{1} y_{1}+x_{2} y_{2}, x_{1} y_{2}-x_{2} y_{1}\right) \quad \text { nodal or focus-focus }
\end{aligned}
$$

with respect to some choice of coordinates near the image point in $B$. An almost toric manifold is a symplectic manifold equipped with an almost toric fibration. A toric fibration is a Lagrangian fibration induced by an effective Hamiltonian torus action.

If a fiber contains $k$ nodal singularities with $k \geq 2$, then the fibration can be perturbed (locally) so that the fiber is replaced by $k$ fibers, each with one nodal singular point. Therefore, for ease of exposition, we assume throughout this paper that a fiber has at most one nodal singular point and call the fiber a nodal fiber.

Remark 2.1. The transverse self-intersection that appears in a nodal fiber is always positive [44]. While Lagrangian planes are not by themselves oriented, any orientation of the base orients these planes (via the Hamiltonian vector fields induced by a basis in the cotangent bundle of the base) thereby giving a well-defined sign to the intersection.

While almost toric fibrations were first defined by Symington [39], interesting examples already existed in the literature on physical integrable systems with two degrees of freedom. In particular, the phase space of the spherical pendulum (cf. [6]) is an almost toric manifold, albeit non-compact and hence outside of the focus of this paper.

We now turn our attention to the geometry that is induced on the base of an almost toric fibration. The rigidity of this geometry and its relation with the topology of the total space yields the primary constraint on almost toric manifolds.

Definition 2.3. The standard integral affine structure $\mathcal{A}_{0}$ on $\mathbb{R}^{n}$ with coordinates $\left(x_{1}, \ldots, x_{n}\right)$ is the lattice $\Lambda_{0}$ in $T \mathbb{R}^{n}$ spanned generated by the vector fields $\frac{\partial}{\partial x_{i}}, i=1, \ldots, n$. 
Definition 2.4. An integral affine structure $\mathcal{A}$ on a manifold $B$ is a lattice $\Lambda$ in its tangent bundle that is locally isomorphic to the standard lattice $\Lambda_{0}$ in $\mathbb{R}^{n}$. In other words, the manifold can be covered with charts $\Phi_{\alpha}: U_{\alpha} \rightarrow \mathbb{R}^{n}$ such that $\Phi_{*} \mathcal{A}=\mathcal{A}_{0}$ and $\left.\Phi_{\alpha} \circ \Phi_{\beta}^{-1}\right|_{U_{\alpha} \cap U_{\beta}} \in \operatorname{Aff}(n, \mathbb{Z})=\operatorname{GL}(n, \mathbb{Z}) \ltimes \mathbb{R}^{n}$. A manifold equipped with such a structure is an integral affine manifold.

A regular Lagrangian fibration induces an integral affine structure on the base via a natural action of the cotangent bundle of the base on the total space. Any point in the base $B$ of a toric fibered four-manifold has a neighborhood that is isomorphic to a neighborhood of a point in the first quadrant $\left(Q, \mathcal{A}_{0}\right) \subset\left(\mathbb{R}^{2}, \mathcal{A}_{0}\right)$ as illustrated by the following example. (In higher dimensions an analogous statement holds.) We call the points in $\partial B$ that correspond to the origin in $\left(Q, \mathcal{A}_{0}\right)$ a vertex and each linear segment on $\partial B$ an edge.

Example 2.1. The base of the local model for a corank two elliptic singularity is $\left(Q, \mathcal{A}_{0}\right)$. Indeed, $\pi:\left(\mathbb{R}^{4}, d x \wedge d y\right) \rightarrow\left(Q, \mathcal{A}_{0}\right)$ is found by choosing coordinates $\left(p_{1}, p_{2}\right)$ on $\mathbb{R}^{2}$ such that $p_{i}=\pi\left(x_{i}^{2}+y_{i}^{2}\right)$. This is then the moment map for the standard torus action on $\mathbb{R}^{4}$. Note that the preimage of a point on the coordinate axes, other than the origin, is a circle of elliptic corank one singular points.

Now consider an almost toric fibration. The image of each nodal fiber is an isolated point on the interior of $B$ which we call a node and view as a singularity in the integral affine structure. Therefore, if $\pi:(M, \omega) \rightarrow(B, \mathcal{A})$ is an almost toric fibration then we understand $\mathcal{A}$ to be the induced structure on all of $B$, namely an integral affine structure with nodes on a manifold with boundary.

If an integral affine structure $\mathcal{A}$ is induced from a toric or almost toric fibration, we call the pair $(B, \mathcal{A})$ a toric base or an almost toric base respectively. In both cases $(B, \mathcal{A})$ is a strong invariant that in dimension four often determines the total space (Corollary 3.1).

With these definitions and observations in mind, we now state the theorems that comprise the diffeomorphism classification.

Theorem 2.1. A closed four-manifold $M$ admits an almost toric fibration with respect to some symplectic structure if and only if $M$ is diffeomorphic to one of the following:

(i) $S^{2} \times S^{2}$ or $\mathbb{C} P^{2} \# n \overline{\mathbb{C} P}^{2}$ with $n \geq 0$,

(ii) $S^{2} \times T^{2}$ or $S^{2} \tilde{\times} T^{2} \# n \overline{\mathbb{C P}}^{2}$ with $n \geq 0$,

(iii) the K3 surface,

(iv) the Enriques surface,

(v) a torus bundle over the torus

$$
M(k ; m, n):=\left\{\left(\begin{array}{ll}
1 & 0 \\
0 & 1
\end{array}\right),\left(\begin{array}{ll}
1 & k \\
0 & 1
\end{array}\right) ; m, n\right\}, k, m, n \in \mathbb{Z}, \text { or }
$$


(vi) a torus bundle over the Klein bottle

$$
N(\delta, k ; a, \epsilon):=\left\{\left(\begin{array}{cc}
1 & \delta \\
0 & -1
\end{array}\right),\left(\begin{array}{cc}
1 & k \\
0 & 1
\end{array}\right) ; a, \epsilon\right\}, \delta, \epsilon \in\{0,1\} \text { and } k, a \in \mathbb{Z} .
$$

Furthermore, Table 1 documents which closed manifolds fiber over which integral affine bases.

The proof of this theorem follows immediately from a collection of results throughout the paper: Theorem 5.2 that specifies the integral affine bases that can occur, Lemmas 6.1 and 6.1 that concern rational surfaces and fibrations over the disk, Lemmas 6.4 and 6.5 that treat the case of sphere bundles over tori and fibrations over the cylinder and Möbius band, Lemmas 6.6-6.8 about K3 and Enriques surfaces and fibrations over $S^{2}$ and $\mathbb{R} P^{2}$, and finally Proposition 6.1 and Theorem 6.4 that cover the cases of torus bundles over the torus and Klein bottle. The manifolds listed in (i)-(iv) of Theorem 2.1 are all easily distinguished by their homology and intersection forms, which also reveal that they are not torus bundles.

In Section 6.4 we prove the following theorem about diffeomorphism equivalences among Lagrangian torus bundles over tori.

Proposition 2.1. Two torus bundles over the torus, $M(k ; m, n)$ and $M\left(k^{\prime} ; m^{\prime}, n^{\prime}\right)$ with $k, k^{\prime} \geq 0$, are diffeomorphic if and only if one of the following holds:

(1) $(k ; m, n)=\left(k^{\prime} ; m^{\prime}, n^{\prime}\right)=(0 ; 0,0)$;

(2) $k n=k^{\prime} n^{\prime}=0,(k ; m, n),\left(k^{\prime} ; m^{\prime}, n^{\prime}\right) \neq(0 ; 0,0)$ and $\operatorname{gcd}(k, m, n)=$ $\operatorname{gcd}\left(k^{\prime}, m^{\prime}, n^{\prime}\right) ;$ or

(3) $k=k^{\prime}>0, n=\epsilon n^{\prime} \neq 0$, and $m \equiv \epsilon m^{\prime}(\bmod k, n)$ where $\epsilon \in\{-1,1\}$.

We refer the reader to Theorem 4(ii) in [41] for the many detailed conditions that dictate which of the torus bundles $N(\delta, k ; a, \epsilon)$ are diffeomorphic. However, it is interesting to note the following:

Proposition 2.2. No manifold admits a Lagrangian torus fibration over both the Klein bottle and the torus.

Proof. Ue [41] showed that the total spaces of the torus bundles $N(\delta, k ; a, \epsilon)$ are diffeomorphic to the total spaces of torus bundles over tori with monodromy matrices $\left(\begin{array}{cc}-1 & x \\ 0 & -1\end{array}\right)$ and $\left(\begin{array}{ll}1 & k \\ 0 & 1\end{array}\right)$ where $x, k \in \mathbb{Z}$. Because $b_{1}=2$, the diffeomorphism classification of these bundles over $T^{2}$ agrees with the isomorphism classification as bundles over $T^{2}$ (Theorem 2 in [34]). Finally, applying the corollary just before Theorem 2 in [34], none of these bundles can be isomorphic to a Lagrangian bundle over the torus. The obstruction to the isomorphism is the fact that $\left(\begin{array}{cc}-1 & x \\ 0 & -1\end{array}\right)$ has trace -2 while the two monodromy matrices for a Lagrangian bundle over $T^{2}$ both have trace 2 and one of them is the identity. 


\section{Total space from the base}

3.1. Toric bases. Delzant's theorem asserts that for a closed toric manifold of dimension $2 n$ the image of the moment map, a polytope in $\mathbb{R}^{n}$, determines the total space, its symplectic structure and the torus action. The Hamiltonian torus action on a toric manifold determines a Lagrangian fibration over an integral affine base that is isomorphic to the moment map image, viewed as a subset of $\left(\mathbb{R}^{n}, \mathcal{A}_{0}\right)$. Accordingly, the Hamiltonian torus action that induces a toric fibration is unique up to reparametrization.

It is important to note that, in contrast to the closed manifold case, different non-compact manifolds can have the same moment map image. Two types of ambiguities arise. First, if the moment map image is not simply connected, then that image does not determine the number of components of the preimage of a value of the moment map. Meanwhile, in dimensions greater than four, having the same simply connected moment map image does not even guarantee that two Lagrangian torus bundles have diffeomorphic total spaces.

Ambiguities concerning the connectedness of fibers and their dimension can be addressed by using the integral affine base $(B, \mathcal{A})$ of a toric fibration rather than its moment map image. Because the set of regular values $B_{0} \subset B$ locally has the structure of the orbit space of a torus action, the fibers are necessarily connected. Meanwhile, the dimension of a fiber can be determined just as in the case of a closed manifold. Indeed, the base is a manifold with corners whose boundary is piecewise linear with respect to the integral affine structure $\mathcal{A}$, and the dimension of a fiber equals the dimension of the linear face that contains the image of the fiber on its interior (except for a zero-dimensional fiber whose image is a point).

Toric bases are easy to recognize. For four-manifolds we have:

Proposition $3.1([39])$. An integral affine two-manifold $(B, \mathcal{A})$, with possibly non-empty boundary, is a toric base if and only it has an integral affine immersion into $\left(\mathbb{R}^{2}, \mathcal{A}_{0}\right)$ and each point in $(B, \mathcal{A})$ has a neighborhood integral affine isomorphic to a neighborhood of a point in $\left(Q, \mathcal{A}_{0}\right)$, the first quadrant of $\left(\mathbb{R}^{2}, \mathcal{A}_{0}\right)$.

Note that this forces $\Phi(\partial B)$ to be the union of linear segments that either have rational slope or are vertical.

Remark 3.1. It is worth pointing out that the choice of an integral affine immersion $\Phi:(B, \mathcal{A}) \rightarrow\left(\mathbb{R}^{2}, \mathcal{A}_{0}\right)$ determines the isotopy subgroups of the Hamiltonian torus action on each submanifold that is the preimage of an edge of $(B, \mathcal{A})$. The base together with these isotropy data is what Orlik and Raymond call a weighted orbit space. The weighted orbit space determines the four-manifold and the action as a smooth torus action [32]. Different 
choices of integral affine immersions, up to translation, correspond to different parameterizations of the torus that is acting.

In the language of almost toric fibrations, we have the following generalization of Delzant's theorem, in dimension four, to non-compact manifolds:

Proposition $3.2\left([\mathbf{2 5}, \mathbf{3 9}]^{2}\right)$. If $(B, \mathcal{A})$ is a toric base of dimension 2 then there is unique symplectic manifold $(M, \omega)$ that admits a unique Lagrangian fibration $\pi:(M, \omega) \rightarrow(B, \mathcal{A})$. The Hamiltonian torus action that induces this Lagrangian fibration is unique up to reparametrization.

To construct a toric fibered four-manifold from its base $(B, \mathcal{A})$, proceed as follows. A choice of an integral affine immersion $\Phi:(B, \mathcal{A}) \rightarrow\left(\mathbb{R}^{2}, \mathcal{A}_{0}\right)$ provides local coordinates $p$ on any neighborhood of $B$ that embeds in $\mathbb{R}^{2}$ via $\Phi$. Consider a toric fibration $\pi^{\prime}:\left(B \times T^{2}, d p \wedge d q\right) \rightarrow(B, \mathcal{A})$ of a manifold with boundary. The preimage in $\left(B \times T^{n}, d p \wedge d q\right)$ of each linear component $L$ of $\Phi(\partial B)$ is fibered by circles that are in the kernel of $\left.\omega\right|_{L}$. Collapsing each of these circles to a point yields $(M, \omega)$ and a toric fibration $\pi:(M, \omega) \rightarrow(B, \mathcal{A})$. (The procedure has been called boundary reduction by the second author $[\mathbf{3 9}]$.) The fact that each vertex of $(B, \mathcal{A})$ is modeled on a neighborhood of the vertex in $\left(Q, \mathcal{A}_{0}\right)$ guarantees that the quotient of $B \times T^{2}$ under all the collapsing maps is a manifold. (Further details can be found in $[\mathbf{3 9}]$ where manifolds with non-empty boundary were included in the discussion. Therefore in [39] the stratification of the base according to the dimension of the preimages was included as part of the data needed to determine the total space.)

Note that there is a symplectic projection $\rho:\left(B \times T^{n}, d p \wedge d q\right) \rightarrow(M, \omega)$ that is a diffeomorphism over the points $x$ of $M$ such that $\pi(x)$ belongs to the interior of $(B, \mathcal{A})$. Indeed, this presentation gives local action-angle coordinates $(p, q)$ on a dense subset of $(M, \omega)$, with $\left(\frac{\partial}{\partial p_{1}}, \ldots \frac{\partial}{\partial p_{n}}\right)$ being a basis for the lattice in the tangent space at any point of $(B, \mathcal{A})$. Furthermore, on the preimage of regular values of $\pi$ the map $\pi$ is the moment map for the free action $t \cdot(p, q)=(p, q+t)$ which extends uniquely to all of $M$. Different choices of integral affine immersion $\Phi:(B, \mathcal{A}) \rightarrow\left(\mathbb{R}^{2}, \mathcal{A}_{0}\right)$ differ only by an element of $\operatorname{Aff}(2, \mathbb{Z})$ and, up to translation, correspond to torus actions differing by reparametrization of the torus.

In higher dimensions, the analogous procedure yields the unique Lagrangian fibration over $(B, \mathcal{A})$ that has a Lagrangian section.

3.2. Integral affine monodromy. As we shall see in Section 3.5, while Proposition 3.2 does not generalize completely to the almost toric case,

\footnotetext{
${ }^{2}$ For higher dimensions, to achieve uniqueness one must require that the induced fibration have a Lagrangian section. Accordingly, Theorem 3.19 of [39] should have included a restriction to dimension four.
} 
it comes close. In many cases the base of an almost toric fibration does determine the total space.

An essential way in which the base $(B, \mathcal{A})$ influences the topology of the total space is by capturing the monodromy. Specifically, the topological monodromy of the torus fibration over the regular values $B_{0} \subset B$ is determined by monodromy in the lattice $\Lambda \subset T B_{0}$ (or, dually, in $\Lambda^{*} \subset T^{*} B_{0}$ ) that defines the integral affine structure $\left.\mathcal{A}\right|_{B_{0}}$.

The integral affine monodromy of an integral affine manifold $B$ is defined analogously to the monodromy of a torus fibration (cf. [15]). Specifically, if $\Lambda$ is the lattice in $T B$, choose a point $b \in B_{0}$, identify $\left(T_{b} B, \Lambda_{b}\right)$ with $\left(\mathbb{R}^{n}, \mathbb{Z}^{n}\right)$ and for each element $\alpha \in \pi_{1}\left(B_{0}, b\right)$ choose a representative $\gamma_{\alpha}: I \rightarrow B_{0}$. The monodromy representation is $\Psi_{B}: \pi_{1}(B, b) \rightarrow \operatorname{Aff}(n, \mathbb{Z})$ where $\Psi_{B}(\alpha)$ is the automorphism of $\left(\mathbb{R}^{n}, \mathbb{Z}^{n}\right)$ such that $\gamma_{\alpha}^{*}(T B, \Lambda)$ is isomorphic to $I \times$ $\left(\mathbb{R}^{n}, \mathbb{Z}^{n}\right) /(0, p) \sim\left(1, \Psi_{B}(\alpha)(p)\right), p \in \mathbb{R}^{n}$. The monodromy is the equivalence class of monodromy representations relative to different points in $B$ and different choices of identification of $T_{b} B$ with $\left(\mathbb{R}^{n}, \mathbb{Z}^{n}\right)$.

The link between the topological and affine monodromies can be seen most easily in local action-angle coordinates $(p, q)$ on a neighborhood of a regular fiber $F_{b}=\pi^{-1}(b)$. The vectors $\frac{\partial}{\partial p_{1}}, \ldots, \frac{\partial}{\partial p_{n}}$ at $b$ form a basis for $\Lambda_{b}$ and the homology classes of integral curves tangent to the vector fields $\left(\frac{\partial}{\partial q_{1}}, \ldots \frac{\partial}{\partial q_{n}}\right)$ on $\pi^{-1}(b)$ represent a basis for $H_{1}\left(F_{b}, \mathbb{Z}\right)$. With respect to these bases, if the topological monodromy of the Lagrangian fibration along a loop $\gamma$ based at $b$ is given by $A \in G L(n, \mathbb{Z})$, then the affine monodromy along $\gamma$ is given by its inverse transpose $\left(A^{-1}\right)^{T}$. This follows immediately from the requirement that the endomorphism of $T_{x} M, x \in F_{b}$, determined by the topological and affine monodromies be symplectic.

3.3. Neighborhood of a node. Henceforth, we restrict our attention to dimension four. The goal of this section is to clarify the extent to which the integral affine structure in a neighborhood of a node, containing just the one singular point, determines the manifold fibering over it.

It is well known that the monodromy around a nodal fiber is, with respect to some basis for the first homology of a regular fiber $F_{b}=\pi^{-1}(b)$,

$$
A_{(1,0)}:=\left(\begin{array}{ll}
1 & 1 \\
0 & 1
\end{array}\right) \text {. }
$$

This has been calculated in the integrable systems setting (e.g. $[\mathbf{6}, \mathbf{1 0}])$ but is also understood to be a property of the underlying smooth torus fibration (cf. [27] or [15]) where it is the monodromy around a singular fiber in a positive Lefschetz fibration.

The reader should note that $A_{(1,0)}$ is a parabolic matrix with eigenvector $\left(\begin{array}{l}1 \\ 0\end{array}\right)$. With respect to an arbitrary basis the monodromy matrix has the form

$$
A_{(a, b)}:=\left(\begin{array}{cc}
1-a b & a^{2} \\
-b^{2} & 1+a b
\end{array}\right)
$$


with eigenvector $\left(\begin{array}{l}a \\ b\end{array}\right)$ for some relatively prime $a, b \in \mathbb{Z}$. Viewing the singular fiber as a regular fiber with a circle pinched to a point, this circle (which represents the vanishing cycle) represents the homology class $(a, b)$.

Meanwhile, the discussion of Section 3.2 implies that the affine monodromy around the image of the nodal fiber is $A_{(-b, a)}$. Therefore the vector $(-b, a)$ is tangent to the one well-defined line that passes through the node. Accordingly we call this line the eigenline through the node.

Knowing the monodromy around an isolated singular point in an affine surface does not completely determine the germ of its neighborhood. In particular, there is an infinite family of isolated singularities around which the monodromy is parabolic. To distinguish between them, remove an eigenray $R$ based at the node from a regular neighborhood $N$ of the node, choose an integral affine immersion $\Phi:(N-R, \mathcal{A}) \rightarrow\left(\mathbb{R}^{2}, \mathcal{A}_{0}\right)$ and count the number of preimages of a generic point in the image. The following lemma, together with the fact that the monodromy around a node is parabolic, implies the uniqueness of the germ of a neighborhood of a node.

Lemma 3.1. Let $N$ be an integral affine open disk that contains exactly one node. Suppose $R$ is a properly embedded ray based at the node $b$. Then any integral affine immersion $\Phi:(N-R, \mathcal{A}) \rightarrow\left(\mathbb{R}^{2}, \mathcal{A}_{0}\right)$ is an embedding.

A proof of this lemma can be found in [38] or Section 9.2 of [39]. It relies on the fact, due to Gromov [17] and Eliashberg [11], that a fillable contact three-manifold is tight. This lemma can also be deduced from the existence of a circle action on a neighborhood of a nodal fiber [44]. Lemma 3.1 allows us to talk about nodes as well-defined singularities in integral affine manifolds without reference to almost toric fibrations.

While nodal fibers occur naturally in certain examples coming from algebraic geometry and integrable systems, these typically do not give a clear picture of the local fibered structure. For an explicit local model consult Section 4.4 of [39].

Given our interest in the symplectic and topological properties of the total space of an almost toric four-manifolds, the following uniqueness properties suffice.

Proposition 3.3. Consider symplectomorphic neighborhoods of a pair of nodal fibers in an almost toric fibration. The symplectomorphism between them can be chosen to be fiber-preserving on the complement of smaller fibered neighborhoods.

The proof of this proposition given in $[\mathbf{3 8}]$ can easily be modified to accommodate multiple singularities on the nodal fiber.

It is important to note that while the germ of a neighborhood of a nodal fiber with a fixed number of singular points is unique up to symplectomorphism, it is not unique up to fiber-preserving symplectomorphism. Indeed, 
$\mathrm{Vu}$ Ngoc $[\mathbf{4 3}]$ has identified a non-trivial invariant that classifies the germs of such neighborhoods up to fiber-preserving symplectomorphism.

3.4. Almost toric bases. Recall that an almost toric base is an integral affine manifold that is the base $(B, \mathcal{A})$ of an almost toric fibration of a manifold (without boundary). The construction of toric manifolds given in Section 3.1 and the normal form for elliptic singular points imply that the image of any such point has a neighborhood that is integral affine isomorphic to a neighborhood of a point in the boundary of $\left(Q, \mathcal{A}_{0}\right)$. Recall that the images of nodal fibers are nodes on the interior of the base. Therefore, since the condition for a fibration to be almost toric is a local one, we have the following characterization of almost toric bases of dimension two:

Proposition 3.4. An integral affine two-manifold, with possibly non-empty boundary, is an almost toric base if and only if each point has a neighborhood that is integral affine isomorphic to a neighborhood of a node or a neighborhood of a point in $\left(Q, \mathcal{A}_{0}\right)$ where $Q$ is the first quadrant in $\mathbb{R}^{2}$.

Propositions 3.2 and 3.3 imply that the neighborhood of any fiber in an almost toric fibration, singular or not, has a neighborhood that can be recovered (up to a variation in the fibration near a nodal fiber) from the base of the fibration. For a more global statement, we appeal to Zung's study of Lagrangian fibrations with topologically stable non-degenerate singularities [45]. An essential invariant therein is the Lagrangian Chern class, an element of the first homology of the base with values in the sheaf of closed basic one-forms (closed one-forms that vanish on vectors tangent to fibers) modulo those forms that arise from contracting the vector fields for a symplectic fiber-preserving circle actions with the symplectic form. (To be precise, this Chern class is actually a relative class in the sense that it is defined relative to a given reference fibration.) From Zung's work we extract the following:

Proposition 3.5 ([45]). In dimension four, an almost toric manifold is determined, up to fiber-preserving symplectomorphism, by its base $(B, \mathcal{A})$, the Lagrangian Chern class and the local structure of the fibered neighborhoods of its nodal fibers.

Note that if the base has the homotopy type of a zero- or one-dimensional manifold, then the Lagrangian Chern class vanishes. Therefore Propositions 3.3 and 3.5 imply the following analog of Proposition 3.2:

Corollary 3.1. If an almost toric base $(B, \mathcal{A})$ of dimension two has the homotopy type of a zero- or one-dimensional manifold, then $(B, \mathcal{A})$ determines the total space of an almost toric fibration up to symplectomorphism.

Even if the base does not determine the total space, one can nonetheless easily extract the Euler characteristic of the total space from the geometry of the base. 
Lemma 3.2. The Euler characteristic of the total space of an almost toric fibration equals the sum of the number of nodes and the number of vertices in the integral affine base.

Proof. The total space can be built up as follows. Start with the union of regular fibers which is a torus bundle over a surface. The set of circle fibers has a neighborhood that is a disjoint union of $D^{3} \times S^{1}$ 's and disk bundles over a torus (if $\partial B$ contains a circle that is geodesic with respect to $\mathcal{A}$ ) that is glued onto the initial manifold on a domain diffeomorphic to either $I \times S^{2} \times S^{1}$ or an $I \times S^{1}$ bundle over the torus, respectively. At this stage one has a manifold with zero Euler characteristic. The total space of the fibration can be completed by gluing in an open ball neighborhood for each point fiber (corank two elliptic singular point) along an $I \times S^{3}$ and a neighborhood of a nodal fiber that deformation retracts to that fiber along a torus bundle over an interval. Thus the Euler characteristic equals the sum of the number of point singularities and the number of nodal fibers.

3.5. Base diagrams and branch moves. In this section we introduce diagrams in $\left(\mathbb{R}^{2}, \mathcal{A}_{0}\right)$ that allow for reconstruction of certain almost toric bases. In light of Corollary 3.1, these can be viewed as a generalization of moment maps.

Definition 3.1. Consider an integral affine surface $(B, \mathcal{A})$ with nodes $\left\{b_{i}\right\}_{i=1}^{k}$. A set of branch curves for $(B, \mathcal{A})$ is a union of disjoint properly embedded curves, $R=\cup\left\{R_{i}\right\}_{i=1}^{k}$, such that each $R_{i}$ has one endpoint at $b_{i}$ and $R_{i} \cap \partial \bar{B}$ is one point (so $B$ cannot be a closed manifold).

Note that since $B$ is locally modeled on $\mathbb{R}^{2}$ on the complement of the nodes, $\bar{B}$ is well defined. An essential feature of branch curves is that whenever $B$ is a disk, there is an immersion of $(B-R, \mathcal{A})$ into $\left(\mathbb{R}^{2}, \mathcal{A}_{0}\right)$. Consequently, whenever the universal cover of $B$ embeds smoothly in $\mathbb{R}^{2}$, there exists an affine immersion into $\left(\mathbb{R}^{2}, \mathcal{A}_{0}\right)$ of a fundamental domain of $(B-R, \mathcal{A})$.

Definition 3.2. Suppose $(B, \mathcal{A})$ is an almost toric base that contains a union of branch curves $R \subset B$ such that there exists an integral affine embedding $\Phi:(B-R, \mathcal{A}) \rightarrow\left(\mathbb{R}^{2}, \mathcal{A}_{0}\right)$. A base diagram of $(B, \mathcal{A})$ with respect to $R$ and $\Phi$ is the image $\Phi(B-R)$ with the following additional data:

(1) an asterisk indicating the location of a node and

(2) heavy dashed lines indicating the portion of $\partial \overline{\Phi(B-R)}$ that corresponds to $R$, i.e., is comprised of points that would map to $R$ under continuous extensions of $\left(\left.\Phi\right|_{U}\right)^{-1}$ where $U$ is any neighborhood on which $\Phi$ is an embedding.

We call a base diagram of a fundamental domain of $(B, \mathcal{A})$ a base diagram for $(B, \mathcal{A})$ if it is adorned with the usual notations indicating how to 
reconstruct $B$ (arrows along edges that should be identified) as in Figure 7 . We distinguish $\partial B$ by using a heavy line.

Example 3.1. Let $U$ be any neighborhood of the origin in $\mathbb{R}^{2}$ and let $R$ be a ray with rational slope $\frac{b}{a}$ based at the origin. (Assume $a, b$ are relatively prime integers.) Then $U-R \subset \mathbb{R}^{2}$ with an asterisk at the origin is a base diagram for a regular neighborhood of a nodal fiber $\pi:(N, \omega) \rightarrow(B, \mathcal{A})$. Furthermore, with respect to coordinates (on the complement of a ray in $(B, \mathcal{A}))$ induced from the base diagram, the affine monodromy around the node is $A_{(a, b)}$.

The next example shows how a base diagram varies depending on the choice of ray that is removed.

Example 3.2. Suppose $\pi:(N, \omega) \rightarrow(B, \mathcal{A})$ is an almost toric fibration of a regular neighborhood of a nodal fiber. Choose a ray $R$ based at the node and integral affine coordinates on the complement of $R$ such that the affine mon-

odromy is $A_{(1,0)}$. If the ray $R$ belongs to a line with slope $\frac{b}{a}$ then there is a projection $\Phi:(N-R, \mathcal{A}) \rightarrow\left(\mathbb{R}^{2}-S, \mathcal{A}_{0}\right)$ where $S$ is the sector bounded by the vectors $(a, b)$ and $(a+b, b)$. This projection will be surjective onto $U-S$ where $U$ is a neighborhood of the origin.

Varying the choice of branch curve constitutes a branch move. Thus there are two ways to vary a base diagram when $B$ is a two-disk: via branch moves and by changing the projection by composing with an element of $\operatorname{Aff}(2, \mathbb{Z})$. (Of course, if $B$ is not a two-disk, then we can also vary the choice of fundamental domain.) While one base that immerses into $\mathbb{R}^{2}$ has many base diagrams, from any one of them one can reconstruct the integral affine base.

\section{Almost toric surgeries}

4.1. Nodal trade. In this section, we describe a surgery operation that changes an almost toric fibration of a symplectic four-manifold into another almost toric fibration of the same symplectic manifold. The essential idea is that one can trade a zero-dimensional singular fiber for a nodal fiber (and vice versa under the appropriate conditions).

We start with an even simpler way to modify almost toric fibrations:

Definition 4.1. Two almost toric bases $\left(B, \mathcal{A}_{i}\right), i=1,2$, are related by a nodal slide if there is a curve $\gamma \subset B$ such that $\left(B-\gamma, \mathcal{A}_{1}\right)$ and $\left(B-\gamma, \mathcal{A}_{2}\right)$ are isomorphic and, for each $i, \gamma$ contains one node of $\left(B, \mathcal{A}_{i}\right)$ and $\gamma$ belongs to the eigenline through that node.

A nodal slide should be thought of as a one-parameter family of almost toric bases in which a node moves in the base along its eigenline. Of course, 
it corresponds to a one-parameter family of almost toric fibrations of one manifold. Exactness of the symplectic structures on the preimage of a neighborhood of $\gamma$ allows us to use Moser's argument to confirm that the symplectic manifolds that fiber over $\left(B, \mathcal{A}_{i}\right), i=1,2$, are symplectomorphic. This perspective on nodal slides makes it easy to find a one-parameter family of almost toric fibrations connecting a fibration with a singular fiber having $k$ nodes to a fibration in which each fiber has only one singular point. In the base, at one extreme one would have a node of multiplicity $k$ and at the other one would have $k$ nodes that live on one line, the eigenline.

If the eigenline through a node intersects the interior of an edge in the base then the limit of nodal slides as the node approaches the edge will result either in changing the topology of the total space to form an orbifold, or else merely a change in the fibration that replaces the nodal fiber with an elliptic singular point of corank two. We call this operation a nodal trade. Zung [45] had observed that this operation could be performed on Lagrangian fibrations and that the one-parameter family connecting the initial and final fibrations appears frequently in integrable systems: it is a Hamiltonian-Hopf bifurcation [42].

As an example of a pair of bases related by a nodal trade, consider the base diagrams shown in Figures 1(a) and (b). These base diagrams define symplectomorphic manifolds.

Indeed, Figure 1(a) is a base diagram corresponding to a toric fibration of the standard symplectic four-ball. Meanwhile, Figure 1(b) is a base diagram for an almost toric four-ball: the preimage of a sufficiently small collar neighborhood of the boundary of the base is an $S^{1} \times D^{3}$, or $D^{4}$ with a one-handle attached; the preimage of the whole base differs by attaching a -1-framed two-handle that is a thickening of the vanishing disk of the nodal fiber, but this two-handle cancels the one-handle yielding $D^{4}$.

Since the base diagram in Figure 1(a) is the limit of a nodal slides of the node in Figure 1(b), we can again invoke Moser's argument to establish that the total spaces fibering over the two bases are symplectomorphic.
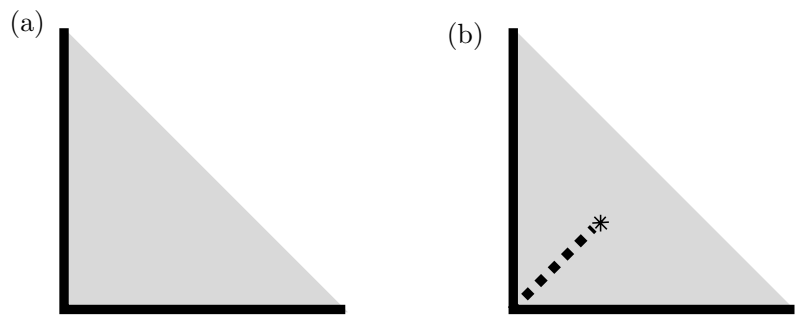

Figure 1. Symplectomorphic four-balls. 
Here is a precise definition:

Definition 4.2. Two almost toric bases $\left(B_{i}, \mathcal{A}_{i}\right), i=1,2$, differ by a nodal trade if each contains an arc $\gamma_{i}$ such that $\left(B_{i}-\gamma_{i}, \mathcal{A}_{i}\right), i=1,2$, are isomorphic, and $\left(B_{1}, \mathcal{A}_{1}\right)$ has one less vertex than $\left(B_{2}, \mathcal{A}_{2}\right)$ does.

The argument that the base diagrams in Figure 1 define symplectomorphic manifolds generalizes in the obvious way to hold for any nodal trade. Therefore,

Theorem 4.1. Two almost toric bases that are related by a nodal trade are symplectomorphic.

4.2. Almost toric blow-up. Blowing up is the simplest way to create a new symplectic manifold from a given one. In dimension four it amounts to removing a symplectic ball and collapsing the circles of the Hopf fibration on the boundary to yield a symplectic sphere of self-intersection -1 . Given a toric manifold, choosing a sufficiently small four-ball to remove, one can blow up so that the resulting manifold is again toric. The effect on a toric base is to cut off a vertex, replacing the vertex by an edge with a vertex at each end. In this case the blow-up point (center of the four-ball to be removed) is the preimage of the vertex.

Within the category of almost toric manifolds $\pi:(M, \omega) \rightarrow(B, \mathcal{A})$ one can also blow-up at points that belong to the preimage of the interior of an edge in $(B, \mathcal{A})$, rather than the preimage of a vertex. This fact was first observed by Zung $[\mathbf{4 5}]$ in the context of Lagrangian fibrations with non-degenerate singularities.

Indeed, consider the base diagrams shown in Figures 2(a) and (b). The first defines a toric fibration of $S^{1} \times D^{3}$ and the second represents the base of an almost toric fibration of $\left(S^{1} \times D^{3}\right) \# \overline{\mathbb{C P}}^{2}$. The -1 -sphere that is introduced in the blow-up can be found in the preimage of an arc connecting the node and the interior of an edge. See Section 5.4 of [39] for more details.

Theorem 3.5 then implies that up to scaling, the action of $\operatorname{Aff}(2, \mathbb{Z})$, or a branch move, Figures 2(a) and (b) completely describe an almost toric surgery that amounts to blowing up the total space. Generalizing in an obvious way, we define:
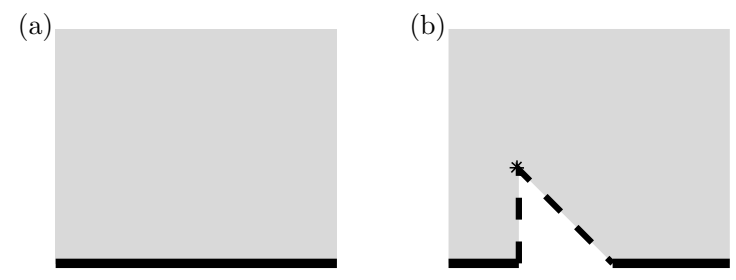

Figure 2. $S^{1} \times D^{3}$ and $\left(S^{1} \times D^{3}\right) \# \overline{\mathbb{C} P}^{2}$. 
Definition 4.3. An almost toric blow-up of an almost toric manifold $\pi:(M, \omega) \rightarrow(B, \mathcal{A})$ is an almost toric fibration $\pi^{\prime}:\left(M \# \overline{\mathbb{C} P}^{2}, \omega^{\prime}\right) \rightarrow\left(B^{\prime}, \mathcal{A}^{\prime}\right)$ such that there is an arc $\gamma^{\prime} \subset B^{\prime}$ based at a node and an integral affine embedding of $\left(B^{\prime}-\gamma^{\prime}, \mathcal{A}^{\prime}\right)$ into $(B, \mathcal{A})$.

\section{Possible bases}

Our goal in this section is to determine what integral affine surfaces with nodes can be the base of a closed almost toric manifold. Theorem 5.2 asserts that the bases are precisely those that appear in Table 1 with the given number of nodes and vertices, and that if the base is a cylinder or Möbius band then the eigenlines of any nodes must be parallel to the boundary.

The first step in proving Theorem 5.2 is to determine that the base $B$ must have non-negative Euler characteristic (Lemma 5.3). The essence of the argument is as follows: on simply connected domains, there are metrics that are compatible in the sense that integral affine lines are geodesic. Then, in a rough sense, by comparison, domains with nodes have non-negative curvature. The curvature contributions cannot be measured using a compatible metric as the presence of non-trivial affine monodromy obstructs the existence of such a metric. However, on disks with nodes we can construct metrics that are inspired by base diagrams, so-called boundary compatible metrics (Definition 5.1). These metrics allow us to bound the total turning angle as the boundary of a disk base is traversed counter-clockwise. The Gauss-Bonnet theorem thereby provides a lower bound on the total curvature of the disk (Lemma 5.2). To rule out surfaces with higher genus or more boundary components occurring as a base, we apply Lemma 5.2 to a fundamental domain in the universal cover.

Definition 5.1. Let $g_{0}$ be the standard metric on $\mathbb{R}^{2}$ compatible with $\mathcal{A}_{0}$. A metric $g$ on an integral affine disk with nodes $(D, \mathcal{A})$ is boundary compatible (with $\mathcal{A}$ ) if a collar neighborhood of the boundary can be covered by a pair of open sets $\{U, V\}$ such that

(1) $U$ is non-empty and simply connected,

(2) $\left.g\right|_{U}=\Phi^{*} g_{0}$ for some integral affine map $\Phi:(U, \mathcal{A}) \rightarrow\left(\mathbb{R}^{2}, \mathcal{A}_{0}\right)$ and

(3) $\partial D \cap V$ is geodesic with respect to both $g$ and $\mathcal{A}$ whenever $V$ is non-empty.

Lemma 5.1. If an integral affine disk with nodes $(D, \mathcal{A})$ has a boundary that contains a segment that is linear (geodesic with respect to $\mathcal{A}$ ) then the disk admits a boundary compatible metric.

Proof. Let $\left\{b_{i}\right\}_{i=1}^{k}$ be the nodes. Choose a set of branch curves $R$ whose endpoints on the boundary all belong to the interior of one linear segment. Let $V$ be a simply connected open subset of $D$ that contains $R$ and such that $V \cap \partial D$ is a linear segment. Let $U$ be an open simply connected subset 
of $D-R$ that covers $\partial D-V$. The simple connectedness of $U$ guarantees the existence of an integral affine immersion $\Phi:(U, \mathcal{A}) \rightarrow\left(\mathbb{R}^{2}, \mathcal{A}_{0}\right)$. Let $g_{U}=$ $\Phi^{*} g_{0}$ and let $g_{V}$ be a metric defined on $V$ such that $\partial D \cap V$ is geodesic with respect to $g_{V}$. Then construct a metric $g$ on $U \cup V$ from $g_{U}$ and $g_{V}$ using a partition of unity subordinate to $\{U, V\}$. Extending $g$ arbitrarily to the rest of the disk, one obtains a boundary compatible metric.

If the boundary of $(D, \mathcal{A})$ admits a boundary compatible metric but contains no linear segments (with respect to $\mathcal{A}$ ), then Definition 5.1 implies $U$ covers $D$, in which case there could not be any nodes in $(D, \mathcal{A})$. Requiring the existence of a linear segment when the monodromy is non-trivial facilitates our upcoming curvature calculations. Note that in all our arguments that involve boundary compatible metrics, there is no loss of generality to assume that $\partial D$ contains a linear segment.

Definition 5.2. Given an integral affine disk with nodes $(D, \mathcal{A})$ consider an integral affine immersion $\Phi:(D-R, \mathcal{A}) \rightarrow\left(\mathbb{R}^{2}, \mathcal{A}_{0}\right)$ for some set of branch curves $R$. A flat model for $(D, \mathcal{A})$ with respect to $R$ and $\Phi$ is the closed flat disk $(\widehat{D}, \hat{g})$ such that $(\operatorname{Int} \widehat{D}, \hat{g})$ and (Int $\left.D-R, \Phi^{*} g_{0}\right)$ are isometric.

Note that if one extends an isometry of $(\operatorname{Int} \widehat{D}, \hat{g})$ onto $\left(\operatorname{Int} D-R, \Phi^{*} g_{0}\right)$ to the boundary of $\widehat{D}$, then the map is a surjection onto $D$ such that points in $R$ other than the nodes each have two preimages.

Lemma 5.2. Let $(D, \mathcal{A})$ be an integral affine disk with nodes equipped with a boundary compatible metric $g$. Then $\int_{D} K_{g} d A \geq 0$, where $K_{g}$ is the Gauss curvature.

Proof. Since $g$ is boundary compatible, there is a pair of sets $\{U, V\}$ and an immersion $\Phi$ as in Definition 5.1 with $\left.g\right|_{U}=\Phi^{*} g_{0}$. If $V$ is empty then $\int_{D} K_{g} d A=0$, so we may assume that $V$ is non-empty. Because $U$ is simply connected we can find a set of branch curves $R=\left\{R_{i}\right\}_{i=1}^{k}$ emanating from the nodes $\left\{b_{i}\right\}_{i=1}^{k}$ that are disjoint from $U$, and hence have their other endpoints on $\partial D \cap V$. (Of course, if there are no nodes, then $R$ is empty.) Replace the affine immersion $\Phi$, defined on $U$, by its extension to all of $D-R$. Let $(\widehat{D}, \hat{g})$ be the flat model for $(D, \mathcal{A})$ with respect to $R$ and $\Phi$. The Gauss-Bonnet Theorem implies

$$
\int_{D} K_{g} d A=2 \pi-\int_{\partial D} \kappa_{g} d s-\beta_{g} \text { and } 0=2 \pi-\int_{\partial \widehat{D}} \kappa_{\hat{g}} d s-\beta_{\hat{g}}
$$

where $\kappa_{g}, \kappa_{\hat{g}}$ are the geodesic curvatures along the smooth parts of $\partial D, \partial \widehat{D}$ and $\beta_{g}, \beta_{\hat{g}}$ are the sums of the turning angles at the vertices of $\partial D, \partial \widehat{D}$. By construction we have

$$
\int_{\partial \widehat{D}} \kappa_{\hat{g}} d s+\beta_{\hat{g}}=\int_{\partial D} \kappa_{g} d s+\beta_{g}+\sum_{i=1}^{k} \theta_{i},
$$




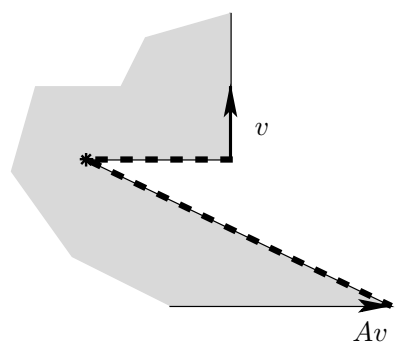

Figure 3. Turning of tangent vectors to the boundary near a node.

where each $\theta_{i}$ is the contribution to the total turning angle along the portion of $\partial \widehat{D}$ introduced by the node $b_{i}$. For instance, Figure 3 shows a base diagram with a branch cut emanating from a node with affine monodromy $A=$ $A_{(1,-1)}$. In this case the node would contribute $\theta=\pi / 4$ to the total turning angle.

The essential fact is that $\theta_{i} \geq 0$ for each $i$. Because these contributions are local, we can assume without loss of generality that there is only one node, which introduces turning $\theta$ as the CCW rotation from $A v$ to $v$. Lemma 3.1 then implies that $-\pi<\theta<\pi$. Meanwhile, by direct calculation $A v \times v \geq 0$ for any $A$ conjugate to $A_{(1,0)}$ and any vector $v \in \mathbb{R}^{2}$. Consequently, $0 \leq \bar{\theta}<$ $p i$, and in particular, $\theta \geq 0$. Therefore $\int_{D} K_{g}=\sum_{i=1}^{k} \theta_{i} \geq 0$.

Remark 5.1. To see that the weak inequality of Lemma 5.2 cannot be replaced by a strict one, consider the base diagram shown in Figure 2(b). It represents a disk with one node such that $A v \times v=0$.

Lemma 5.3. If $(B, \mathcal{A})$ is the base of an almost toric fibration of a closed four-manifold then $\chi(B) \geq 0$.

Note that this lemma was observed by Zung [45] without proof.

Proof. Because the signs of the Euler characteristics of a surface and its double cover are the same, it suffices to work with bases that are orientable.

Ignoring the case in which the base $B$ is a sphere (and hence $\chi(B)>0$ ), we consider a fundamental domain $(\widetilde{D}, \widetilde{\mathcal{A}})$ in the universal cover $(\widetilde{B}, \widetilde{\mathcal{A}})$ of $(B, \mathcal{A})$.

Since the only angles that are well defined with respect to an affine structure are multiples of $\pi$, we follow Benzecri [5] and choose the fundamental domain so that the internal angle at any vertex introduced in the universal cover is zero or $\pm \pi$. For instance, to form the fundamental domain for a surface of genus zero with four boundary components, we can cut it open along three curves $\gamma_{1}, \gamma_{2}$ and $\gamma_{3}$ as shown in Figure 4. Similarly, we can 


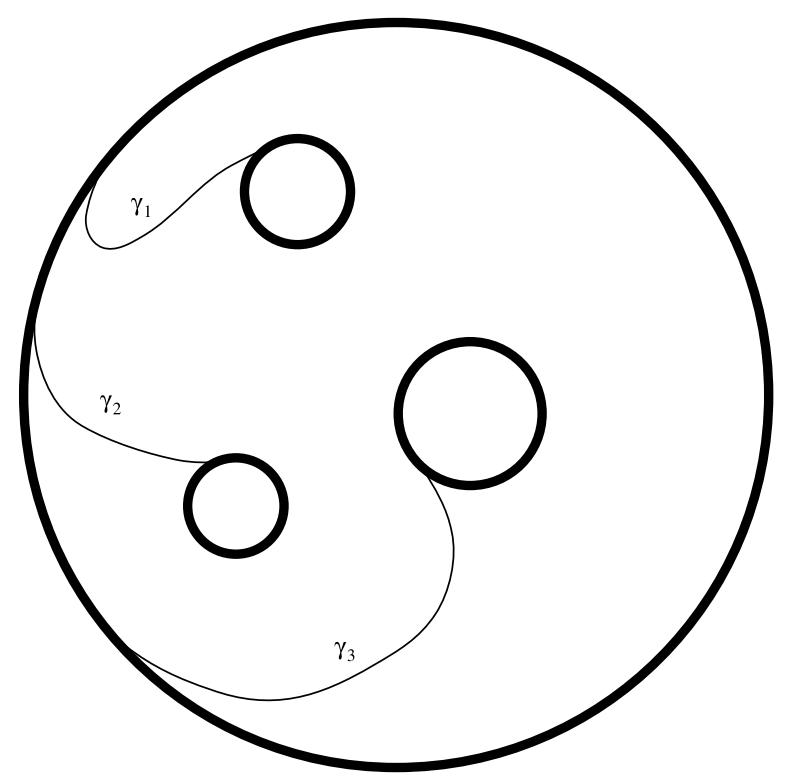

Figure 4. Fundamental domain for a genus zero surface with four boundary components.

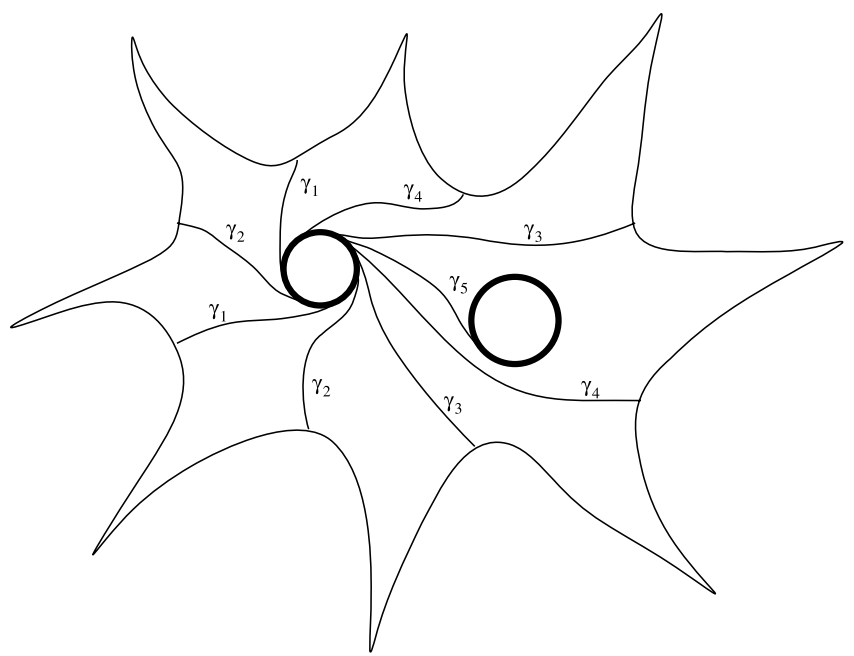

Figure 5. Fundamental domain for a genus two surface with two boundary components.

form the fundamental domain for a surface of genus two with two boundary components as suggested by Figure 5.

The constraints on the topology of $B$ come from the geometry of $(\widetilde{D}, \widetilde{\mathcal{A}})$. Indeed, choosing a metric $\tilde{g}$ on $\widetilde{D}$ that is boundary compatible with $\widetilde{\mathcal{A}}$ 
(after having modified the boundary as needed), the Gauss-Bonnet Theorem implies

$$
2 \pi-\int_{\partial \widetilde{D}} \kappa_{\tilde{g}} d s-\beta_{\tilde{g}} \geq 0
$$

since $\int_{\widetilde{D}} K_{\tilde{g}} d A \geq 0$ by Lemma 5.2 .

To estimate $\int_{\partial \widetilde{D}} \kappa_{\tilde{g}} d s$, note that because $\partial B$ (if non-empty) is piecewise linear, all contributions to the geodesic curvature along the smooth part of $\partial \widetilde{D}$ come from pairs of arcs that each project to one arc in $B$. Naming such a pair $\gamma_{i}, \gamma_{i}^{\prime}$, their images in $\mathbb{R}^{2}$ under the developing map differ by an element of $\operatorname{Aff}(2, \mathbb{Z})$. Specifically, for some orientation preserving element $\Psi \in \operatorname{Aff}(2, \mathbb{Z}), \gamma_{i}^{\prime}=-\Psi\left(\gamma_{i}\right)$. Since elements of $\operatorname{Aff}(2, \mathbb{Z})$ preserve the integer part of $\frac{\theta}{\pi}$ for any angle $\theta$, we have

$$
\left|\int_{\gamma_{i}} \kappa_{\tilde{g}} d s+\int_{\gamma_{i}^{\prime}} \kappa_{\tilde{g}} d s\right|<\pi
$$

for each such pair.

Let $d$ be the genus of the base $B$ and let $m$ be the number of boundary components. We now consider two cases separately: $\partial B$ empty and $\partial B$ nonempty. Recall that we have assumed either $m \geq 1$ or $d \geq 2$.

If $\partial B$ is empty, we can choose a fundamental domain in which all but two of the vertices contribute $\pi$ to the total turning angle and two of them contribute zero. (This satisfies the requirement that the sum of the internal angles of the fundamental domain must equal $2 \pi$.) Then

$$
\beta_{\tilde{g}}=(4 d-2) \pi
$$

and

$$
\left|\int_{\partial \widetilde{D}} \kappa_{\tilde{g}} d s\right|<2 \pi d
$$

in which case inequality (5.5) implies $d=1$.

Suppose now that $\partial B$ is non-empty. The local convexity of $\partial B$ implies that the contributions to $\beta_{\tilde{g}}$ at any vertices of the fundamental domain that project to vertices of $\partial B$ are positive. Meanwhile, the contributions at the other vertices can be calculated exactly - thanks to the turning angle at each being a multiple of $\pi$. Specifically, constructing the fundamental domain in analogy with Figures 4 and 5, we calculate

$$
\beta_{\tilde{g}} \geq 2 \pi(2 d+m-1)
$$

and

$$
\left|\int_{\partial \widetilde{D}} \kappa_{\tilde{g}} d s\right|<\pi(2 d+m-1) .
$$


Since the current assumptions is $m>0$, these inequalities imply that $d=0$ and $m=2$.

In each of the above cases $\chi(B) \geq 0$.

To get more detailed information about the integral affine structure on the almost toric bases in question we need the following standard fact about matrices that encode the monodromy around a node:

Theorem 5.1 ([30]). Suppose $\left\{A_{i}\right\}_{i=1}^{k}$ is a set of matrices in $\mathrm{SL}(2, \mathbb{Z})$, each conjugate to $\left(\begin{array}{ll}1 & 1 \\ 0 & 1\end{array}\right)$. If $\Pi_{i=1}^{k} A_{i}=I$ then $k=12 n$ and there is a finite sequence of elementary transformations that yields the product $\Pi_{i=1}^{k} A_{i}^{\prime}=I$ such that $A_{i}^{\prime}=\left(\begin{array}{ll}1 & 1 \\ 0 & 1\end{array}\right)$ if $i$ is even and $A_{i}^{\prime}=\left(\begin{array}{cc}1 & 0 \\ -1 & 1\end{array}\right)$ if $i$ is odd.

Note that an elementary transformation on a cyclicly ordered set of matrices is either

$$
\begin{aligned}
T_{i} & :\left\{A_{1}, \ldots, A_{i-1}, A_{i}, A_{i+1}, A_{i+2}, \ldots, A_{k}\right\} \\
& \rightarrow\left\{A_{1}, \ldots, A_{i-1}, A_{i} A_{i+1} A_{i}^{-1}, A_{i}, A_{i+2}, \ldots, A_{k}\right\}
\end{aligned}
$$

for some $i$, or its inverse $T_{i}^{-1}$. The relevance of the elementary transforms and Theorem 5.1 follows from:

Observation 5.1. Given an integral affine disk with nodes $(D, \mathcal{A})$, a choice of branch curves $R=\cup_{i=1}^{k} R_{i}$ and an immersion $\Phi:(D-R, \mathcal{A}) \rightarrow\left(\mathbb{R}^{2}, \mathcal{A}_{0}\right)$ determine a representation of the affine monodromy in $\mathrm{SL}(2, \mathbb{Z})$. In particular, if the branch curves are indexed so that their intersections with the boundary give an ordered set of points, say $\left\{x_{1}, x_{2}, \ldots x_{k}\right\}$ agreeing with the orientation of the boundary, then the monodromy along the boundary is $A_{1} A_{2} \ldots A_{k}$ where $A_{i}$ is the monodromy around the node $b_{i}$. The elementary transformation $T_{i}$ then corresponds to replacing the branch curve $R_{i}$ by a branch curve from $b_{i}$ to $x_{i}^{\prime}$ where $x_{i}^{\prime}$ is between $x_{i+1}$ and $x_{i+2}$. Accordingly, we call a change in branch curves corresponding to $T_{i}$ or $T_{i}^{-1}$ an elementary branch move.

Lemma 5.4. Let $(D, \mathcal{A})$ be an affine disk. If the monodromy around the boundary is trivial then there are $12 n$ nodes on the interior of $(D, \mathcal{A})$. Furthermore, for any boundary compatible metric $g$,

$$
\int_{D} K_{g} d A=2 \pi n
$$

Proof. Theorem 5.1 implies that the number of nodes must be a multiple of 12. We proceed as in the proof of Lemma 5.2, choosing a set of branch curves $R=\cup_{i=1}^{k} R_{i}$ and flat model $(\widehat{D}, \hat{g})$. However in this case, if there are nodes, we appeal to Theorem 5.1, and choose the branch curves so that the monodromy $A_{i}$ across each curve $R_{i}$ is $\left(\begin{array}{ll}1 & 1 \\ 0 & 1\end{array}\right)$ if $i$ is even and $\left(\begin{array}{cc}1 & 0 \\ -1 & 1\end{array}\right)$ if $i$ is odd. A direct calculation then shows $\sum_{i=1}^{k} \theta_{i}=2 \pi \frac{k}{12}=2 \pi n$. (It is easiest to 
check this on the vector $(1,0)$ but it is independent of the choice of vector since the total monodromy is trivial.) As in the proof of Lemma 5.2 we have that $\int_{D} K_{g}=\sum_{i=1}^{k} \theta_{i}$ thereby proving the result.

Theorem 5.2. Suppose $(B, \mathcal{A})$ is the base of an almost toric fibration of a closed four-manifold. Then $(B, \mathcal{A})$ must be one of the following:

- a disk with any number of nodes and vertices;

- a cylinder or Möbius band with no vertices and any number of nodes, all of whose eigenlines are parallel to $\partial B$ which is linear; or

- a closed surface with $12 \chi(B)$ nodes.

Proof. An easy way to construct an almost toric manifold whose base is a disk with $k$ nodes and $v$ vertices is to start with the moment map image of $\mathbb{C} P^{2} \#(k+v-3) \overline{\mathbb{C P}}^{2}$ (which is a polygon with $k+v$ vertices) and then perform $k$ nodal trades.

Suppose that $(B, \mathcal{A})$ is a sphere. Cover $(B, \mathcal{A})$ with two disks, $\left(D_{1}, \mathcal{A}\right)$ and $\left(D_{2}, \mathcal{A}\right)$, such that $D_{1} \cap D_{2}=\partial D_{1}=\partial D_{2}$. Assume that $D_{2}$ contains all of the nodes. Then $\left(D_{1}, \mathcal{A}\right)$ admits a flat metric compatible with $\mathcal{A}$ and it extends across $\left(D_{2}, \mathcal{A}\right)$ as a boundary compatible metric $g$. The GaussBonnet Theorem then implies that the total curvature on $D_{2}$ is $4 \pi$, whereby Lemma 5.4 forces there to be 24 nodes. (Consequently, if $B=\mathbb{R} P^{2}$ then $(B, \mathcal{A})$ must have 12 nodes.)

If $B$ is a torus, a priori there could be non-trivial monodromy along generators of $\pi_{1}(B)$ that is balanced by monodromy around nodes $\cup_{i=1}^{k} b_{i}$. This possibility, however, is precluded by the Theorem 2 of [27]. Specifically, Matsumoto proved that one can choose the generators of the fundamental group of the base of an elliptic surface so the monodromy along each generator is trivial. Since almost toric fibrations over closed bases are diffeomorphic to elliptic surfaces, and the monodromy matrices of the elliptic surface are the inverse transposes of the integral affine monodromy matrices of the corresponding almost toric fibration, Matsumoto's theorem applies. Therefore we can proceed as for a sphere, constructing a compatible flat metric on the complement of the interior of a disk that contains all the nodes. Then $\chi(B)=0$ implies that the curvature on that disk is zero, and hence by Lemma 5.4 the disk must contain no nodes.

Now assume that $B$ is a cylinder and that there are no vertices on the boundary, performing nodal trades if necessary. The monodromy around the nodes is quite restricted: Lemma 5.5 below implies that we can choose a monodromy representation in which the monodromy around all the nodes the same, i.e., all the nodes belong to a disk in which there is a well-defined line - the eigenline through the nodes. It remains to check that this line is parallel to the components of $\partial B$ which in turn are parallel to each other. (Recall that the boundary is geodesic because the total space of the fibration 
is a closed manifold.) With the eigenlines parallel to the boundary there is no way to trade any of the nodes for a vertex on the boundary.

For the monodromy presentation choose a base point $b \in B-\cup_{i=1}^{k} b_{i}$, a basis for $T_{b} B$ and simple loops $\gamma_{i}, 1 \leq i \leq k$, based at $b$ such that

(1) each $\gamma_{i}$ winds around exactly one node $b_{i}$, positively oriented as the boundary of the disk it bounds and

(2) the affine monodromy along each $\gamma_{i}$ is $A_{(1,0)}$.

Now let $\eta_{1}, \eta_{2}$ be loops based at $b$ that generate $\pi_{1}(B)$ and are such that

$$
\eta_{2}^{-1} \eta_{1}=\gamma_{k} \cdots \gamma_{1}
$$

so that there are no nodes between $\eta_{1}$ and one component of $\partial B$ and no nodes between $\eta_{2}$ and the other component of $\partial B$. Since the affine monodromy along each $\gamma_{i}$ is $A_{(1,0)}$, Equation (5.13) implies that the affine monodromy along $\eta_{2}^{-1} \eta_{1}$ is $A_{(1,0)}^{k}$. It is easy to check that this can happen only if the monodromy along both $\eta_{1}$ and $\eta_{2}$ is a power of $A_{(1,0)}$ whenever $k \neq 0$. Geometrically, this means that the boundary components are parallel to the common eigenline of the nodes. Note that it also implies $\partial B$ cannot have any vertices.

If there are no nodes (the case $k=0$ ) then the monodromy along $\eta_{1}$ is the same as along $\eta_{2}$. Furthermore, this monodromy must be conjugate to a power of $A_{(1,0)}$ since a tangent vector to either linear boundary component must be invariant under the monodromy. (This is most easily seen in a base diagram of a fundamental domain.) Therefore the boundary components are parallel to each other.

The corresponding statement for a Klein bottle base $B$ follows because it must be covered by an integral affine cylinder. Any vertices on $(B, \mathcal{A})$ would lift to vertices in the cover, and any eigenlines not parallel to the boundary would lift to eigenlines not parallel to the boundary of the cover.

Lemma 5.5. Suppose $(B, \mathcal{A})$ is a cylinder or Möbius band whose boundary is linear with respect to $\mathcal{A}$. Assume that there are nodes in the affine structure $\mathcal{A}$. Then there is a presentation of the affine monodromy such that the monodromy around each node is $A_{(1,0)}$, i.e., all the eigenlines are parallel.

Proof. Consider first the case of a cylinder base. Choose a fundamental domain $(\widetilde{D}, \tilde{\mathcal{A}}) \subset(\widetilde{B}, \tilde{\mathcal{A}})$ and a boundary compatible metric $\tilde{g}$. Then Equations (5.9) and (5.10) imply, with $d=0$ and $m=2$, that $\beta_{\tilde{g}} \geq 2 \pi$ and $\left|\int_{\gamma} \kappa_{\tilde{g}} d s\right|<\pi$. With these constraints the Gauss-Bonnet Theorem forces $\int_{\widetilde{D}} K_{\widetilde{g}} d A<\pi$. Our proof by contradiction amounts to construction of a boundary compatible metric that violates this bound.

If there is only one node, then the conclusion of the lemma is trivial. Therefore, let $\left\{b_{i}\right\}_{i=1}^{k}, k \geq 2$, be the nodes indexed so that the monodromy around $b_{2}$ is not the same as around $b_{1}$. Assume, without loss of generality, 
that $\partial \widetilde{D}$ contains at least four linear segments, one pair of which (say $L_{1}, L_{2}$ ) determines the other. Choose a set of branch curves $R=\cup_{i=1}^{k} R_{i}$ such that $R_{i} \cap \partial \widetilde{D} \in L_{i}$ for $i=1,2$ and an immersion $\Phi:(\widetilde{D}-R, \mathcal{A}) \rightarrow\left(\mathbb{R}^{2}, \mathcal{A}_{0}\right)$ such that the monodromy around $b_{1}$ is $A_{(1,0)}$. Then following the proof of Lemma 5.1, we construct a boundary compatible metric $\tilde{g}$ on $(\widetilde{D}, \widetilde{A})$ whose total curvature is $\int K_{\tilde{g}} d A=\sum_{i=1}^{k} \theta_{i}$ where $\theta_{i}$ measures the curvature contribution of $b_{i}$ as in the proof of Lemma 5.2. Since $\theta_{i} \in[0, \pi)$ for each $i$ (as shown in that proof), we only need to verify that we can choose the fundamental domain so that $\theta_{1}+\theta_{2} \geq \pi$.

Referring to Figure 3 and suppressing indices, we note that

$$
\tan \theta=\frac{A v \times v}{A v \cdot v}
$$

where $A$ is the monodromy around a node. With $A=A_{(p, q)}$ and $v=(x, y)$ we calculate

$$
\begin{aligned}
A v \times v & =(q x-p y)^{2}, \\
A v \cdot v & =(1-p q) x^{2}+\left(p^{2}-q^{2}\right) x y+(1+p q) y^{2} .
\end{aligned}
$$

Allowing the affine lengths of $L_{1}, L_{2}$ to be sufficiently small, we can choose the fundamental domain so that the vectors $v_{1}, v_{2}$ have any direction we want. Recalling that we have chosen $A_{1}=A_{(1,0)}$, Equations (5.15) and (5.16) imply $\theta_{1}$ is maximized by taking $v_{1}=(-1,2)$ in which case $\tan \theta_{1}=4 / 3$. For $A_{2}=A_{(p, q)}$ we can assume $q \geq 1$ since $q=0$ would make the eigenvectors parallel and the vector $(p, q)$ is defined only up to sign. Furthermore, we can choose (without loss of generality) $p$ to be positive and arbitrarily large since it is defined only mod $q$. (To change the value of $p$ we can modify our choice of affine immersion $\Phi$ in a way that causes $A_{2}$ to be conjugated by a power of $A_{(1,0)}$ - and therefore leaves $A_{1}$ unchanged.)

Having chosen $v_{1}=(-1,2)$ the bound $\theta_{1}+\theta_{2}<\pi$ will violated if

$$
\tan \theta_{2}<0
$$

and

$$
\left|\tan \theta_{2}\right| \leq \tan \theta_{1}=\frac{4}{3}
$$

We do this by choosing $v_{2}=(p+2, q)$ so that

$$
A_{2} v_{2} \times v_{2}=4 q^{2}
$$

and

$$
A_{2} v_{2} \cdot v_{2}=(1-2 q) p^{2}+4(1-q) p+\left(4+q^{2}-2 q^{3}\right) .
$$

Choosing $p$ large enough we have that $\tan \theta_{2}$ is negative and as close to zero as we like. 


\section{Classification}

\subsection{Rational surfaces.}

6.1.1. Existence and equivalences. It is well known that a closed fourmanifold admits a toric fibration if and only if the manifold is diffeomorphic to a rational surface, i.e., $\mathbb{C} P^{2} \# m \overline{\mathbb{C} P}^{2}$ or $\left(S^{2} \times S^{2}\right) \# n \overline{\mathbb{C} P}^{2}$, and that $\left(S^{2} \times S^{2}\right) \# \overline{\mathbb{C} P}^{2}$ is diffeomorphic to $\mathbb{C} P^{2} \# 2 \overline{\mathbb{C} P}^{2}$ (cf. [2] or [12]). The base of a toric fibration of a rational surface is integral affine isomorphic to the moment map image in $\mathbb{R}^{2}$ for a torus action inducing the fibration. Note that the number of vertices in the integral affine base $(B, \mathcal{A})$ (or moment map image) of a toric fibration of $\mathbb{C} P^{2} \# m \overline{\mathbb{C P}}^{2}$ or $S^{2} \times S^{2}$ is $m+3$ or 4 , respectively.

Since any vertex can be traded for a node by performing a nodal trade, we have:

Lemma 6.1. $\mathbb{C} P^{2} \# m \overline{\mathbb{C P}}^{2}$ and $S^{2} \times S^{2}$ admit almost toric fibrations over integral affine disks with $n$ nodes and $k$ vertices provided $n+k=m+3$ or $n+k=4$, respectively.

Remark 6.1. One nice feature of almost toric fibrations of rational surfaces is that they provide families of fibrations that interpolate between different toric fibrations of a manifold while maintaining the cohomology class of the symplectic form. (See [39] Section 6.2.) This is always true for Hirzebruch surfaces which are diffeomorphic to either $S^{2} \times S^{2}$ or $\mathbb{C} P^{2} \# \overline{\mathbb{C P}}^{2}$. We conjecture that such interpolating almost toric fibrations exist between toric fibrations of any rational surface.

6.1.2. Completeness. The only manifolds that admit almost toric fibrations over the disk are the rational surfaces. More specifically,

Theorem 6.1. Suppose $\pi:(M, \omega) \rightarrow(B, \mathcal{A})$ is a closed almost toric manifold whose base is a disk. Then there is a symplectic structure $\omega^{\prime}$, deformation equivalent to $\omega$, such that $\left(M, \omega^{\prime}\right)$ admits a toric fibration.

The essential idea of the proof of Theorem 6.1 is simple: just slide the nodes on the interior of $B$ along eigenrays until they hit the boundary, thereby performing the inverse of a nodal trade. However, the almost toric base in question may be such that:

(1) there is no set of disjoint eigenrays connecting the nodes to the boundary (along which to slide the nodes) or

(2) sliding a node all the way to the boundary might produce a change in topology by creating an orbifold singular point.

The first issue is addressed by Lemma 6.2 which allows us to assume that all nodes are close enough to the boundary for there to be a "good" set of branch curves. Specifically, the branch curves can be chosen so that each 
one belongs either to an eigenline or to a neighborhood of a boundary point in which the eigenline through the node is parallel to the base. As explained in Section 4.2, such a node is the result of an almost toric blow-up.

We resolve the second issue by providing (in the proof of Lemma 6.3) an algorithm to appropriately modify an almost toric disk base without changing the topology of the total space it defines.

For simplicity of exposition, we assume that there are no vertices on the boundary. (If not, we start by trading all vertices for nodes.)

To begin, we indicate a set of data on a base diagram that defines the topology of the total space.

Lemma 6.2. Suppose $\pi:(M, \omega) \rightarrow(D, \mathcal{A})$ is a closed almost toric manifold whose base is a disk with no vertices on the boundary. Suppose $V=$ $\Phi(D-R) \subset\left(\mathbb{R}^{2}, \mathcal{A}_{0}\right)$ is the domain of a base diagram, where $R=\cup_{i=1}^{k} R_{i}$ is a set of branch curves. Let $u_{i}, i=1, \ldots, k$ be the inward-pointing primitive integral vectors normal to the connected components of $\partial \mathrm{V}$, indexed so that they rotate non-negatively (counterclockwise). Then the set of vectors $\left\{u_{1}, \ldots u_{k}\right\}$, up to cyclic permutation and the action of $\mathrm{GL}(n, \mathbb{Z})$, determines the diffeomorphism type of $M$.

Note that $\Phi$ is an embedding since $\partial D$ is locally convex. Also, each vector $u_{i}$ really should be viewed as a covector defining the corresponding connected component of $\partial V$. We follow the convention that $u_{i-1}$ and $u_{i}$ are normal to the connected components of $\partial V$ on either side of the cut created by $R_{i}$.

Remark 6.2. For the reader familiar with complex algebraic toric varieties, the vectors $u_{i}$ define a complete fan. The toric variety defined by the fan will in general have orbifold singularities and hence not be diffeomorphic to $M$. It will fail to be smooth precisely when $u_{i} \times u_{i+1}>1$ for some $i$.

Proof. Because $(D, \mathcal{A})$ has no vertices its boundary is geodesic with respect to $\mathcal{A}$. Therefore $M$ is the boundary reduction (along one line) of a symplectic manifold $\left(M^{\prime}, \omega^{\prime}\right)$ that is a smooth Lefschetz fibration over a disk. As such, the diffeomorphism type of $M^{\prime}$ are completely determined by the monodromy. The only additional data needed to determine $M$ are the homology class of a regular fiber that gets collapsed during the boundary reduction (the collapsing class). This homology class is well defined only with respect to an arc in the base that runs from the image of a regular fiber to the boundary.

The ordered set of vectors $\left\{u_{1}, \ldots, u_{k}\right\}$ defines the monodromy because for each pair $\left\{u_{i}, u_{i+1}\right\}$ there is a unique matrix $A_{i}$ conjugate to $A_{(1,0)}$ such that $A_{i} u_{i}=u_{i-1}(\bmod k)$. Furthermore, the collapsing class can be defined by $u_{i}$ for any $i$ as follows: choose an embedded arc $\gamma$ connecting a point $\Phi(b)$ on the interior of $V$ and a point in the connected component of $\partial V$ defined by $u_{i}$. Viewing $u_{i}$ as a covector in $T^{*} \mathbb{R}^{2}$ and pulling back via $\Phi$ we 
get a covector in $T_{b}^{*} D$. The collapsing class with respect to $\Phi^{-1}(\gamma)$ is then the element of $H_{1}\left(F_{b}, \mathbb{Z}\right)$ represented by integral curves of the vector field $X$ such that $\omega(X, \cdot)=\Phi^{*} u_{i}$. (Here $F_{b}$ is the regular torus fiber over the point $b \in D$ and $\omega$ is the symplectic structure defined by the almost toric fibration.)

Since cyclicly permuting the vectors $u_{1}, \ldots, u_{k}$ has no effect on the monodromy presentation or the collapsing class it also has no effect on the topology. Furthermore, changing the vectors by applying an element of $\operatorname{GL}(2, \mathbb{Z})$ amounts only to changing the isomorphism between $H_{1}\left(F_{b}, \mathbb{Z}\right)$ and $\mathbb{Z}^{2}$.

Remark 6.3. Unless the monodromy along the boundary of the base (with no vertices) is trivial, the collapsing class is determined by the monodromy. Indeed, $A_{1} A_{2} \cdots A_{k} u_{k}=u_{k}$ and, unless $A_{1} A_{2} \cdots A_{k}=I$, the primitive integral vector $u_{k}$ is unique up to sign. In contrast, if $A_{1} A_{2} \cdots A_{k}=I$ then any vector could determine a collapsing class with respect to a fixed arc.

Theorem 6.1 implies the diffeomorphism type of the total space is independent of this choice of vector. Since the boundary is geodesic, the proof of Lemma 5.4 forces the number of nodes to be 12 and therefore the total space is diffeomorphic to the elliptic surface $E(1)$. Accordingly, the possibility of choosing any vector to determine the collapsing class whenever $A_{1} A_{2} \cdots A_{k}=I$ reflects the very large diffeomorphism group of $E(1)$.

A natural question is what sequences of vectors $\left\{u_{1}, \ldots, u_{k}\right\}$ can be the normal vectors to the connected components of $\partial V$ where $V=\Phi(D-R)$ as above? The primary constraint is that

$$
A_{i} u_{i}=u_{i-1}
$$

for each $i(\bmod k)$ where $A_{i}$ is some matrix conjugate to $A_{(1,0)}$. The action of $A_{i}$ on any vector $v$ can be rewritten in terms of its eigenvector $e_{i}$ as

$$
A_{i} v=v-\left(v \times e_{i}\right) e_{i} .
$$

Therefore, the constraint can be rewritten as

$$
u_{i-1}=u_{i}-\left(u_{i} \times e_{i}\right) e_{i}
$$

for some primitive integral vector $e_{i}$. The only other constraint on $\left\{u_{1}, \ldots u_{k}\right\}$ is that the vectors rotate exactly once around the origin. This motivates

Definition 6.1. An ordered set of primitive integral vectors $\left(u_{1}, \ldots, u_{k}\right)$ is a defining set for a closed almost toric manifold fibering over a disk if for each $i$ there is an integer $n_{i}$ and primitive integral vector $e_{i}$ such that

(1) $u_{i} \times e_{i}=n_{i}$,

(2) $u_{i}-u_{i-1}=n_{i} e_{i}$ where the indices are understood $\bmod k$,

(3) $u_{j} \neq u_{1}$ for some $j \neq 1$ and

(4) if $u_{m}=u_{1}$ for some $m$, then either $u_{i}=u_{1}$ for all $i \leq m$ or else $u_{i}=u_{1}$ for all $i \geq m$. 
Note that $u_{i-1} \times u_{i}=n_{i}^{2}$ for each $i(\bmod k)$. Furthermore, the definition of $e_{i}$ is such that $n_{i} \geq 0$ for all $i$.

Recalling Remark 6.2 and the discussion after Theorem 6.1, for each $i$ we must have either $n_{i}=1$ (so we can slide node $b_{i}$ into the boundary) or $n_{i}=0$ (so $b_{i}$ is the result of blowing up).

Lemma 6.3. Suppose $\left\{u_{1}, \ldots, u_{k}\right\}$ is a defining set for a closed symplectic four-manifold $(M, \omega)$. There is a sequence of elementary branch moves (defined in Observation 5.1) that yields a new defining set $\left\{u_{1}^{\prime}, \ldots u_{k}^{\prime}\right\}$ such that $u_{i-1}^{\prime} \times u_{i}^{\prime}=\left(n_{i}^{\prime}\right)^{2} \in\{0,1\}$ for all $i$.

Proof. The defining set determines a corresponding set of monodromy matrices $\left\{A_{1}, A_{2}, \ldots A_{k}\right\}$. The elementary branch move corresponding to the elementary transformation $T_{j}$ causes $u_{j}$ to be replaced by $A_{j} u_{j+1}$, leaving the other vectors $u_{i}, i \neq j$, unchanged.

Let $\tau_{j}$ be the induced action on the integers $\left\{n_{1}, n_{2}, \ldots n_{k}\right\}$. Then

$$
\begin{aligned}
\tau_{j}\left(n_{i}\right) & =n_{i} \quad \text { if } i \neq j, j+1, \\
\tau_{j}\left(n_{j}\right) & =\sqrt{u_{j-1} \times A_{j} u_{j+1}} \\
& =\sqrt{A_{j} u_{j} \times A_{j} u_{j+1}} \\
& =\sqrt{u_{j} \times u_{j+1}}=n_{j+1}
\end{aligned}
$$

and

$$
\begin{aligned}
\tau_{j}\left(n_{j+1}\right) & =\sqrt{A_{j} u_{j+1} \times u_{j+1}} \\
& =\sqrt{\left(u_{j+1}-\left(u_{j+1} \times e_{j}\right) e_{j}\right) \times u_{j+1}} \\
& =\sqrt{\left(u_{j+1} \times e_{j}\right)^{2}}=\left|u_{j+1} \times e_{j}\right|,
\end{aligned}
$$

where the second equality follows from Equation (6.22). Therefore, performing a sequence of elementary branch moves corresponding to $T_{j+m} \cdots T_{j+1} T_{j}$ has the effect, via $\tau_{j+m} \cdots \tau_{j+1} \tau_{j}$, of removing $n_{j}$ from the set and inserting $\left|u_{j+m+1} \times e_{j}\right|$.

Assume, without loss of generality, that $n_{1}=N \geq n_{i}$ for all $i$. Also assume that $N \geq 2$ (for otherwise our initial sequence would satisfy the conclusion of the lemma).

If $\left|u_{i} \times e_{1}\right|<N$ for some $i$, then performing a branch move that corresponds to $T_{i-1} \cdots T_{2} T_{1}$ removes $n_{1}=N$ and replaces it by a strictly smaller non-negative integer. Redefining $u_{1}$ we could apply the same argument repeatedly. Therefore the only obstruction to achieving $n_{i} \in\{0,1\}$ for all $i$ would be if at some stage $\left|u_{i} \times e_{1}\right| \geq N$ for all $i$. Assume this is true.

Let $f_{1}$ be a primitive integral vector such that $e_{1} \times f_{1}=1$. Then we can write any vector $v$ as a linear combination of $e_{1}, f_{1}$ where $e_{1} \times v$ gives the $f_{1}$ coefficient and $v \times f_{1}$ gives the $e_{1}$ coefficient. Furthermore, the condition 


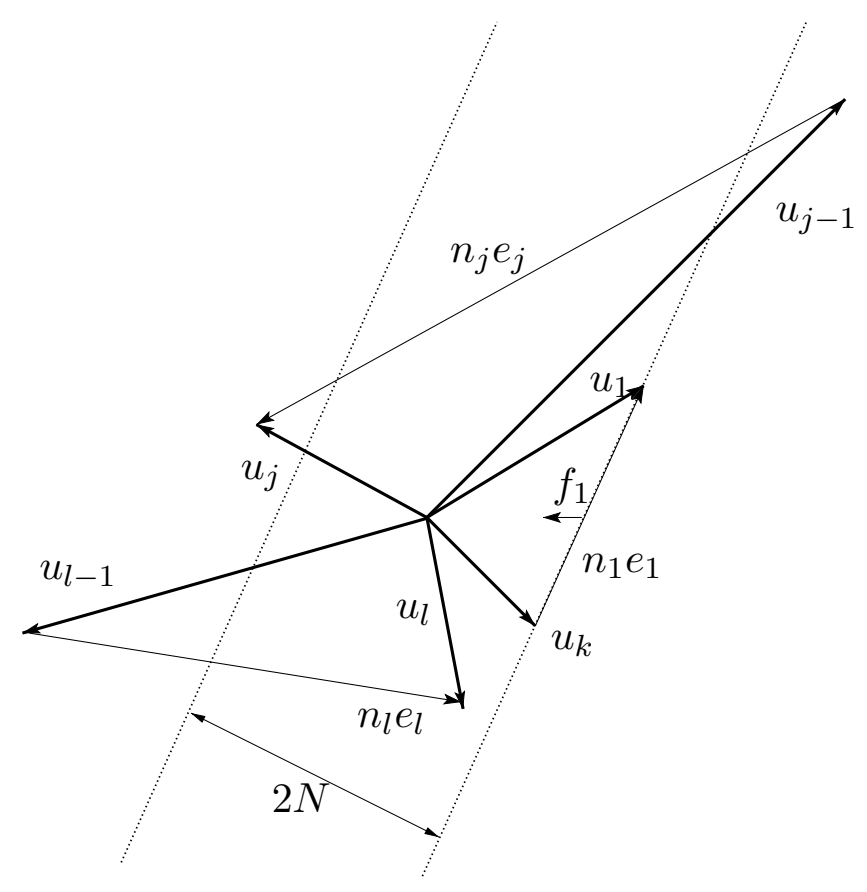

Figure 6. Vectors of a defining set.

that $\left|u_{i} \times e_{1}\right| \geq N$ for all $i$ implies that the endpoints of all the vectors $u_{i}$ based at the origin must fall outside the open domain between by the lines parameterized by $t \in \mathbb{R}$ as $\pm N f_{1}+t e_{1}$ in $\mathbb{R}^{2}$. (See Figure 6 in which $u_{l}$ fails this condition.)

Since the equation $e_{1} \times f_{1}=1$ defines $f_{1}$ modulo an integer multiple of $e_{1}$, we can choose $f_{1}$ so that $u_{k} \times f_{1}<0$ and $u_{1} \times f_{1} \geq 0$. (See Figure 6.) Furthermore, we can strengthen the last inequality to $u_{1} \times f_{1}>0$ because $u_{1} \times f_{1}=0$ and the primitivity of $u_{1}$ would imply $u_{1}= \pm f_{1}$, which would contradict the assumption that $N=u_{1} \times e_{1} \geq 2$.

Because the angle between $u_{i-1}$ and $u_{i}$ is less than $\pi$ for any $i$, there must be some minimal $j$ such that $e_{1} \times u_{j} \geq N$. Writing $e_{j}=x e_{1}+y f_{1}$, we have $y>0$ because $e_{1} \times e_{j}>0$ by construction. (Again, see Figure 6.) Meanwhile,

$$
\begin{aligned}
n_{j} & =u_{j-1} \times e_{j} \\
& =x\left(u_{j-1} \times e_{1}\right)+y\left(u_{j-1} \times f_{1}\right),
\end{aligned}
$$

but $u_{j-1} \times e_{1} \geq N$ and $u_{j-1} \times f_{1}>0$ so the only way to have $n_{j} \leq N$ is if $x<0$. (Note that $x$ and $y$ are both integers.) Furthermore,

$$
\begin{aligned}
n_{j} & =u_{j} \times e_{j} \\
& =x\left(u_{j} \times e_{1}\right)+y\left(u_{j} \times f_{1}\right)
\end{aligned}
$$


where $u_{j} \times e_{1} \leq-N$. With $y>0$ and $x<0$, the constraint that $n_{j} \leq N$ forces $u_{j} \times f_{1} \leq 0$. Since $u_{j} \times f_{1}=0$ would imply $u_{j}=f_{1}$ and thereby $N=1$, we find $u_{j} \times f_{1}<0$, i.e., the $e_{1}$ component of $u_{j}$ must be negative.

By symmetry, the same argument for $u_{l-1}, u_{l}$, where $l$ is the maximal index for which $e_{1} \times u_{l-1} \geq N$, would show that the $e_{1}$ component of $u_{l-1}$ must be positive.

Since both of these conditions on the $e_{1}$ components of $u_{j}$ and $u_{l-1}$ cannot be met, the assumption that $N \geq 2$ must have been false.

Proof of Theorem 6.1. Assume, without loss of generality, that the almost toric fibration is over a disk that has no vertices. Let $\left\{u_{1}, \ldots u_{k}\right\}$ be a defining set of vectors arising from a particular base diagram. Invoking Lemma 6.3 we can, by varying the base diagram without changing the fibration, find a new defining set $\left\{u_{1}^{\prime}, \ldots, u_{k}^{\prime}\right\}$ such that $n_{i}^{2}=u_{i-1}^{\prime} \times u_{i}^{\prime} \in\{0,1\}$ for all $i$. In this base diagram the branch curves need not be linear.

Now, allowing the fibration and symplectic structure to vary, we construct a new almost toric base $\left(D, \mathcal{A}^{\prime}\right)$ that defines the same smooth four-manifold but has a more amenable base diagram. Indeed, letting $l \leq k$ be the number of distinct vectors in the defining set, we construct the base diagram as follows:

(1) Choose a convex polygon such that $\left\{u_{1}^{\prime}, \ldots, u_{k}^{\prime}\right\}$ is a set of inward pointing normal vectors that rotate non-negatively counterclockwise. This will be a polygon with $l$ sides.

(2) For each $i$ such that $u_{i}^{\prime} \neq u_{i-1}^{\prime}(\bmod k)$, place a dotted line segment $\eta_{i}^{\prime}$ in the polygon so that it has one endpoint at the vertex between the sides with normal vectors $u_{i-1}^{\prime}, u_{i}^{\prime}$ and has $e_{i}^{\prime}$ as a tangent vector. Do this so that the $\eta_{i}^{\prime}$ are all disjoint. After placing an asterisk at the interior endpoint of each $\eta_{i}^{\prime}$, this will be the base diagram for an almost toric manifold.

(3) For each $j$ such that $u_{j}^{\prime}=u_{j-1}^{\prime}$, perform an almost toric blow-up on the edge defined by $u_{j}$.

By construction we can now slide all nodes to the boundary, perform $k-l$ almost toric blowdowns, and then perform $k-l$ toric blow-ups. The result will be a toric fibration of $\left(M, \omega^{\prime}\right)$ for some symplectic structure $\omega^{\prime}$.

To see that $\omega^{\prime}$ and $\omega$ must be deformation equivalent note that one could interpolate between the initial base diagram and the final base diagram via a one parameter family of base diagrams. Accordingly one can find a one parameter family of fibrations interpolating between the initial and final ones.

Lemma 3.2 then allows us to deduce the diffeomorphism type of the total space of a toric fibration over the disk from the sum of the numbers of nodes and vertices unless the sum equals four, in which case we only know that $M$ 

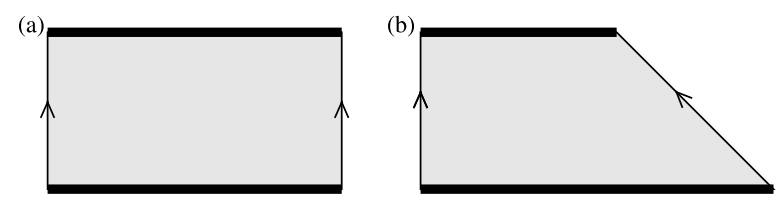

Figure 7. Sphere bundles over tori.

must be a sphere bundle over a sphere - either $S^{2} \times S^{2}$ or $\mathbb{C} P^{2} \# \overline{\mathbb{C} P}^{2}$. The two sphere bundles can be easily distinguished by the moment map image (or base) of a toric fibration (cf. [39] or [2]). It would be interesting to know if there is a quick way to distinguish these sphere bundles from the base of an almost toric fibration, without carrying out the algorithm in the proof of Theorem 6.1 that leads one to a toric fibration.

\subsection{Sphere bundles over the torus.}

6.2.1. Existence and equivalence. Up to diffeomorphism there are two sphere bundles over the torus, $S^{2} \times T^{2}$ and the non-trivial bundle $S^{2} \tilde{\times} T^{2}$.

We can construct almost toric fibrations of these manifolds over a cylinder as follows. Start with a toric fibration of $\mathbb{R} \times S^{1} \times S^{2}$ over $\mathbb{R} \times[-1,1]$ given by $\pi(p, q, \phi, \theta)=(p, \sin \phi)$ where $p$ is a linear coordinate on $\mathbb{R}, q$ is an angular coordinate of period 1 on $S^{1}$, and $-\frac{\pi}{2} \leq \phi \leq \frac{\pi}{2}, 0 \leq \theta<2 \pi$ are angular coordinates on $S^{2}$. This is a toric fibration with respect to the symplectic form $\omega=d p \wedge d q+d(\sin \phi) \wedge d(\theta / 2 \pi)$.

To get $T^{2} \times S^{2}$, quotient by an action of $\mathbb{Z}$ whose generator is the symplectomorphism that sends $(p, q, \phi, \theta)$ to $(p+a, q, \phi, \theta), a \neq 0$, which induces the integral affine transformation $\left(I ;\left(\begin{array}{c}a \\ 0\end{array}\right)\right)$ on the base. To get a non-trivial sphere bundle over the torus we can choose the generator of $\mathbb{Z}$ to be the symplectomorphism that sends $(p, q, \phi, \theta)$ to $(p-\sin \phi+a, q, \phi, q+\theta / 2 \pi)$, $a \neq 0$, which induces the integral affine transformation $\left(\left(\begin{array}{cc}1 & -1 \\ 0 & 1\end{array}\right) ;\left(\begin{array}{l}a \\ 0\end{array}\right)\right)$. Base diagrams for such almost toric fibrations are given in Figures 7(a) and (b), respectively. The monodromy along a curve parallel to the boundary in the base is given by

$$
\left(\begin{array}{ll}
1 & k \\
0 & 1
\end{array}\right)
$$

with $k=0$ and -1 , respectively, if the curve is oriented to the right.

Similarly, one can construct fibrations in which the monodromy is $k$ for any $k \in \mathbb{Z}$, in which case the slope of the right-hand edge in the base diagram is $\frac{-1}{k}$. Applying the methods in Section 7 of [39] one can determine that the self-intersection of the preimage of the lower edge would then equal $k$. Therefore, the bundle is trivial or non-trivial according to whether $k$ is even or odd, respectively. 
Sphere bundles over the torus also admit almost toric fibrations over a Möbius band, again as quotients by $\mathbb{Z}$ of a toric fibration of $\mathbb{R} \times S^{1} \times S^{2}$ over $\mathbb{R} \times[-1,1]$. Indeed, precompose the aforementioned generators of the $\mathbb{Z}$ action by the rotation $(p, q, \phi, \theta) \mapsto(p, q,-\phi,-\theta)$. Because a rotation is isotopic to the identity this does not change the resulting total space. Precomposing by this rotation does, however, have the effect of changing the integral affine transformations to $\left(\left(\begin{array}{cc}1 & 0 \\ 0 & -1\end{array}\right) ;\left(\begin{array}{l}a \\ 0\end{array}\right)\right)$ and $\left(\left(\begin{array}{cc}1 & 1 \\ 0 & -1\end{array}\right) ;\left(\begin{array}{l}a \\ 0\end{array}\right)\right)$, respectively. The corresponding base diagrams differ from those in Figure 7 only by reversing the arrow on the right-hand edge.

Blow-ups of these sphere bundles also admit almost toric fibrations. Indeed, one can perform an arbitrary number of (sufficiently small) almosttoric blow-ups, each one introducing one node in the base.

Similar to sphere bundles over the sphere, blow-ups of the trivial and nontrivial sphere bundles over the torus are diffeomorphic. This can be proved in terms of base diagrams by making a branch move that changes which component of the boundary is intersected by the branch curve emanating from a given node.

Summarizing, we have proved:

Lemma 6.4. Blow-ups of the trivial and non-trivial sphere bundle over the torus, $\left(S^{2} \times T^{2}\right) \# n \overline{\mathbb{C P}}^{2}$ and $\left(S^{2} \tilde{\times} T^{2}\right) \# n \overline{\mathbb{C P}}^{2}$, admit almost toric fibrations over both integral affine cylinders and Möbius bands that have $n$ nodes. Among these manifolds, $\left(S^{2} \times T^{2}\right) \# n \overline{\mathbb{C} P}^{2}$ and $\left(S^{2} \tilde{\times} T^{2}\right) \# n \overline{\mathbb{C P}}^{2}$ are diffeomorphic for each $n \geq 1$.

6.2.2. Completeness. Suppose $\pi:(M, \omega) \rightarrow(B, \mathcal{A})$ is an almost toric fibration in which the base $B$ is homeomorphic to a cylinder or a Möbius band. Then Theorem 5.2 implies that, after blowing down, the integral affine base must be isomorphic to a quotient of a strip $\{(x, y) \mid a \leq y \leq b\} \subset$ $\left(\mathbb{R}^{2}, \mathcal{A}_{0}\right)$ by an action of $\mathbb{Z}$. The monodromy for such a quotient must be of the form $\left(\begin{array}{ll}1 & k \\ 0 & \pm 1\end{array}\right)$ for some $k \in \mathbb{Z}$ in order to have the boundary invariant. By Corollary 3.1 we know that these integral affine cylinders and Möbius bands determine the total spaces uniquely. Noting that the preimage of a vertical cross section of the base is a copy of $S^{1} \times S^{2}$, we see that the total space must be a sphere bundle over a torus. Thus,

Lemma 6.5. The only closed four-manifolds that admit an almost toric fibration over a cylinder or Möbius band are sphere bundles over tori and blow-ups of these.

\subsection{K3 and Enriques surface.}

6.3.1. Existence and completeness. A complex surface is a $K 3$ surface if it is simply connected and it supports a non-vanishing holomorphic twoform $\Omega$. Many K3 surfaces admit a holomorphic fibration with generic fibers 
being tori. Topologically, a generic holomorphic fibration of a K3 surface is a genus one Lefschetz fibration with 24 singular fibers (cf. [15]). All K3 surfaces are diffeomorphic so in the smooth category we refer to "the K3 surface."

The existence of almost toric fibrations of the K3 surface is well known in mirror symmetry where such fibrations underly generic special Lagrangian fibrations (cf. [20]). Any K3 surface, as a complex manifold, is hyperkähler and therefore equipped with a two-sphere of complex structures with corresponding symplectic forms that are the Kähler forms. (In complex dimension two the only closed hyperkähler manifolds are the K3 surfaces and complex tori $-T^{4}$ equipped with differing complex structures.) A hyperkähler rotation (cf. [22]) applied to a holomorphically fibered hyperkähler manifold transforms the holomorphic fibration into a special Lagrangian fibration, i.e., a Lagrangian fibration that is also adapted to the complex structure in the sense that $\operatorname{Im}(\Omega)=0$ on the fibers for some choice of non-vanishing holomorphic two-form $\Omega$. Therefore, a hyperkähler rotation of a generic holomorphically fibered K3 surface yields an almost toric fibered K3. These general statements imply:

Lemma 6.6. The K3 surface admits an almost toric fibration over the sphere with 24 nodes.

To get a better understanding of such fibrations, we detail a construction of almost toric fibered K3 surfaces that are based on the well-known fact that the K3 surface, or $E(2)$, is the fiber sum of two copies of the elliptic surface $E(1)=\mathbb{C} P^{2} \# 9 \overline{\mathbb{C} P}^{2}$ which fibers over $\mathbb{C} P^{1}$ with 12 nodal fibers (cf. [15]).

(1) Choose two toric fibrations of $E(1)=\mathbb{C} P^{2} \# 9 \overline{\mathbb{C} P}^{2}$.

(2) The base diagram of each has 12 vertices. Perform nodal trades at all of the vertices. This yields two almost toric manifolds, each with a smooth symplectic torus of self-intersection zero fibering over the boundary of the base. This almost toric fibration has 12 singular fibers that are Lagrangian and hence are not the singular fibers of a symplectic or holomorphic fibration. However, the preimage of the boundary is a symplectic torus that can be viewed as a regular fiber of a Lefschetz fibration with symplectic fibers.

(3) Symplectic sum the two almost toric copies of $E(1)$ along these symplectic tori. In the base this amounts to joining the two bases along their boundaries. (The symplectic sum requires that the symplectic tori along which one sums have the same area. This corresponds to the boundaries of the integral affine bases of the almost toric fibered $E(1)$ 's being integral affine isomorphic. Both constraints are purely a matter of scaling.) 
An Enriques surface is a complex manifold that is a $\mathbb{Z}_{2}$ quotient of a $\mathrm{K} 3$ surface. In our construction of an almost toric fibered K3 surface there is no obstruction to making the integral affine base have $\mathbb{Z}_{2}$ symmetry. If the base has such symmetry then one can take a fiber-preserving $\mathbb{Z}_{2}$ quotient of the K3 surface to get an almost toric manifold fibering over $\mathbb{R} P^{2}$. One might expect that the quotient manifold is diffeomorphic to an Enriques surface even though the base of the fibration is $\mathbb{R} P^{2}$ rather than the usual $\mathbb{C} P^{1}$ base of a holomorphic fibration. However, have no way to ensure that the $\mathbb{Z}_{2}$ quotient in question supports a complex structure. Therefore, we describe a construction of an almost toric fibration over $\mathbb{R} P^{2}$ that allows us to see, in the smooth category, that the total space is an Enriques surface. Because all Enriques surfaces are diffeomorphic we refer to "the Enriques surface" when discussing the underlying smooth manifold.

Lemma 6.7. The Enriques surface admits an almost toric fibration over $\mathbb{R} P^{2}$.

Before proving this lemma we review some pertinent background. The (smooth) $\log$ transform ${ }^{3}$ is a surgery on a four-manifold in which one removes a neighborhood of a regular torus fiber and glues it back in via a diffeomorphism of the boundary (cf. [15]). When performed on a genus one Lefschetz fibration, the effect of the log transform on the total space depends only on an integer, the multiplicity: it produces a multiple fiber of multiplicity $p$, namely a fiber $f_{p}$ whose homology class satisfies $p\left[f_{p}\right]=[f]$ where $f$ is a regular fiber.

Crucial to our argument is the fact that $E(1)_{2,2}$ is the Enriques surface (cf. [15]). Here $E(1)_{2,2}$ denotes the manifold obtained from $E(1)=$ $\mathbb{C} P^{2} \# 9 \overline{\mathbb{C} P}^{2}$ by performing two smooth multiplicity two log transforms along fibers of a genus one Lefschetz fibration, which is the smooth ana$\log$ of an elliptic fibration of the elliptic surface $E(1)$.

Proof. Consider an almost toric fibration of the elliptic surface $E(1)$ such as used in constructing an almost toric fibered K3 surface, i.e., one whose base $B$ has 12 nodes and a boundary that is linear with respect to the integral affine structure. Now remove a neighborhood of $\partial B$ that is fibered by geodesics parallel to the boundary (with respect to the affine structure) and replace it with a Möbius band also fibered by geodesics parallel to the boundary. The result is an integral affine $\mathbb{R} P^{2}$ with 12 nodes. This integral affine surgery on the base corresponds to a surgery on the total space in which we remove a neighborhood of the torus $T$ that is the preimage of $\partial B$ and then glue in an almost toric fibration over an open Möbius band, i.e., the product of $S^{1}$ and a three-manifold that is

\footnotetext{
${ }^{3}$ The log transform is traditionally an operation on complex algebraic surfaces (cf. [16]). The smooth log transform is the induced surgery on the underlying smooth manifold.
} 
the circle bundle over a Möbius band whose total space is orientable. This latter three-manifold can be obtained from a solid torus by performing two Dehn surgeries of multiplicity two along circles parallel to the core (cf. [21]). Since the product of the identity map (on $S^{1}$ ) and a Dehn surgery of multiplicity $k$ (on a solid torus) is a log transform of multiplicity $k$, we have that the surgery used to construct the total space is equivalent to two log transforms of multiplicity two. Since the product of the $S^{1}$ factor and circles parallel to the core of the solid torus correspond to fiber tori of a genus one Lefschetz fibration of $E(1)$, we have that the resulting manifold is indeed $E(1)_{2,2}$, and hence the Enriques surface.

Lemma 6.8. The K3 surface and the Enriques surface are the only closed manifolds that admit almost toric fibrations over the sphere and $\mathbb{R} P^{2}$, respectively.

Proof. By Theorem 5.2 any integral affine structure on the sphere or $\mathbb{R} P^{2}$ must have 24 or 12 nodes, respectively. Therefore, as a smooth fibration, an almost toric fibration over $S^{2}$ is equivalent to a genus one Lefschetz fibration with 24 singular fibers, and hence diffeomorphic to a K3 surface (cf. [15]). Similarly, the smooth fibration underlying any almost toric fibration over $\mathbb{R} P^{2}$ is a genus one Lefschetz fibration over $\mathbb{R} P^{2}$. Our proof of Lemma 6.7 can be carried out without modification in the smooth category - the integral affine structure on the base was used merely as a guide - thereby implying that any almost toric fibration over $\mathbb{R} P^{2}$ has the Enriques surface as its total space.

\subsection{Torus bundles over the torus.}

6.4.1. Existence and completeness. By Theorem 5.2, almost toric fibrations over the torus have no singular fibers and hence are regular fibrations, or Lagrangian torus bundles. The torus bundle $M\{A, B ; m, n\}$ can be constructed as follows. Let $\alpha, \beta$ be generators of the fundamental group of the base and consider the unique torus bundle with section whose monodromy representation maps $\alpha$ to $A$ and $\beta$ to $B$. Next, remove a product neighborhood $T^{2} \times D^{2}$ of a fiber. Let $\lambda_{1}, \lambda_{2}$ and $\mu$ be curves on the boundary $T^{3}$ whose homology classes generate $H_{1}\left(T^{3}, \mathbb{Z}\right)$ and such that $\mu$ is a meridional curve. Now glue the $T^{2} \times D^{2}$ back in by a map that takes $\mu$ to a curve in the class $[\mu]+m\left[\lambda_{1}\right]+n\left[\lambda_{2}\right]$.

The integral affine structure on the base torus, arising because the fibers of the bundle are Lagrangian, restricts the possibilities for monodromy.

Proposition 6.1 ([29]). A torus bundle over the torus admits a symplectic structure with respect to which the bundle is Lagrangian if and only if, for 
some choice of framing of the fiber, it has the form

$$
M(k ; m, n):=M\left\{\left(\begin{array}{ll}
1 & 0 \\
0 & 1
\end{array}\right),\left(\begin{array}{cc}
1 & k \\
0 & 1
\end{array}\right) ; m, n\right\}
$$

with $k, m, n \in \mathbb{Z}$ and $k \geq 0$.

Note that Geiges [13] identified which torus bundles over tori admit a symplectic structure with respect to which the underlying fibration is Lagrangian. In particular, Geiges [13] observed that the torus bundles $M(k ; m, n)$ fall into three classes depending on whether the first Betti number equals 4 (when $k=m=n-0$ ), 3 (when $k=0$ and $(m, n) \neq 0$ or $k>0$ and $n=0$ ) or 2 (when $k>0$ and $n \neq 0$ ). If $b_{1}=4$ then the manifold is $T^{2} \times T^{2}$ and admits a Kähler structure. Those that have $b_{1}=3$ admit complex structures but no Kähler structures and are the Kodaira manifolds that were among the first examples of non-Kähler symplectic manifolds [40]. The Lagrangian torus bundles over tori that have $b_{1}=2$ admit no complex structures. In particular, this implies that the hyperelliptic surfaces (the complex torus bundles over tori that have $b_{1}=2$ ) do not admit regular Lagrangian fibrations.

6.4.2. Equivalences. Sakamoto and Fukuhara have classified torus bundles over tori up to bundle isomorphism and global diffeomorphism in [34]. For the convenience of the reader we summarize, in our notation, their results that are relevant to Lagrangian torus bundles:

Proposition 6.2 ([34, p. 313]). $H_{1}(M(k ; m, n))=\mathbb{Z}^{2} \oplus\left(\mathbb{Z}^{2} / K\right)$ where $K$ is the subgroup of $\mathbb{Z}^{2}$ generated by $\left(\begin{array}{c}m \\ n\end{array}\right)$ and $\left(\begin{array}{l}\lambda \\ 0\end{array}\right)$.

Theorem 6.2 ([34, p. 318]). If $b_{1} \neq 3$ then the bundle isomorphism and diffeomorphism classifications coincide.

Note that $b_{1}=3$ if and only if either $k=0$ and $(m, n) \neq(0,0)$ or $k \neq 0$ and $n=0$.

Theorem 6.3 ([34, p. 319]). The bundles $M\{A, I ; m, n\}$ and $M\left\{A^{\prime}, I ; m^{\prime}\right.$, $\left.n^{\prime}\right\}$, where $I$ is the identity matrix, are isomorphic if an only if, for some $P \in \mathrm{GL}(2, \mathbb{Z})$,

$$
P A^{\prime} P^{-1}=A \text { or } P A^{\prime} P^{-1}=A^{-1},
$$

and

$$
\left(\begin{array}{c}
m \\
n
\end{array}\right)-P\left(\begin{array}{c}
m^{\prime} \\
n^{\prime}
\end{array}\right) \text { is in the span of the columns of } A-I .
$$

Proposition 6.3 ([34, p. 321]). $M(k ; m, 0)$ with $(k, m) \neq(0,0)$ is diffeomorphic to $M(d ; 0,0)$ where $d=\operatorname{gcd}(k, m)$.

Sakamoto and Fukuhara only claim homeomorphism equivalence, but the maps used are all smooth. 
Proof of Proposition 2.1. The bundles $M(k ; m, n)$ with $k=m=n=0$, $k n=0$ and $(k, m, n) \neq(0,0,0)$, and $k n \neq 0$ listed in Proposition 2.1 are mutually non-diffeomorphic as their first Betti numbers are 4, 3 and 2 , respectively. After noting that the only bundle with $k=m=n=0$ has total space $T^{4}$, we consider the equivalences within each of the other types separately.

If $n=0$ and $(k, m) \neq(0,0)$ then Proposition 6.3 implies $M(k ; m, n)$ is diffeomorphic to $M(\operatorname{gcd}(k, m, n) ; 0,0)$ since $\operatorname{gcd}(k, m, n)=\operatorname{gcd}(k, m)$ when $n=0$. Suppose $k=0$ and $(m, n) \neq(0,0)$. Then, because $A=I$, the

matrix $P$ in Theorem 6.3 can be chosen to satisfy $\left(\begin{array}{c}m \\ n\end{array}\right)=P\left(\begin{array}{c}\operatorname{gcd}(m, n) \\ 0\end{array}\right)$. Then Proposition 6.3 implies $M(k ; m, n)$ is diffeomorphic to $M(k ; \operatorname{gcd}(m, n), 0)$ which in turn is diffeomorphic to $M(\operatorname{gcd}(k, m, n) ; 0,0)$ because $k=0$.

Theorem 6.3 implies that $|k|$ is a bundle isomorphism invariant of $M(k ; m, n)$, so our assumption that $k, k^{\prime}>0$ implies $k=k^{\prime}$. If $k n \neq 0$ then the matrices that conjugate with $A=\left(\begin{array}{ll}1 & k \\ 0 & 1\end{array}\right)$ are precisely those of the form $P= \pm\left(\begin{array}{ll}1 & p \\ 0 & 1\end{array}\right)$ for some $p \in \mathbb{Z}$. Applying Theorem 6.3 we find that $M(k ; m, n)$ and $M\left(k ; m^{\prime}, n^{\prime}\right)$ are isomorphic if and only if they satisfy the conditions of Proposition 2.1(3). Theorem 6.2 then implies that these conditions also specify the diffeomorphism equivalences.

\subsection{Torus bundles over the Klein bottle.}

6.5.1. Existence and completeness. We denote a torus bundle over the Klein bottle $K$ by $N\{A, B ; a, b\}$ where $A$ is the monodromy along an orientation reversing loop $\alpha$ in $K$ and $B$ is the monodromy along an orientation preserving, non-separating loop $\beta$ in $K$ such that $\alpha$ and $\beta$ intersect once transversely. Consequently, $\alpha \beta \alpha^{-1} \beta$ is null homotopic in $K$ and $A B=B^{-1} A$. The Euler class is defined just as for torus bundles over tori.

Theorem 6.4. A torus bundle over the Klein bottle admits a symplectic structure with respect to which the bundle is Lagrangian if and only if, for some framing of the fiber, it has the form

$$
N(\delta, \lambda ; a, \epsilon):=N\left\{\left(\begin{array}{cc}
1 & \delta \\
0 & -1
\end{array}\right),\left(\begin{array}{cc}
1 & k \\
0 & 1
\end{array}\right) ; a, \epsilon\right\}
$$

for some choice of $\delta, \epsilon \in\{0,1\}$ and $k, a \in \mathbb{Z}$.

Proof. The fundamental group of a torus bundle over the Klein bottle has a presentation with generators $\alpha, \beta, l, h$ and relations

$$
[l, h]=1, \alpha(l, h) \alpha^{-1}=(l, h) A, \beta(l, h) \beta^{-1}=(l, h) B, \alpha \beta \alpha^{-1} \beta=l^{a} h^{b} .
$$

(See $[\mathbf{4 1}]$.) Abelianizing, one finds that $b_{1}=3-\operatorname{rk}(A-I, B-I)$. 
Note that $b_{1}=3$ only if the $2 \times 4$ matrix $(A-I, B-I)$ is identically zero, which is impossible because $\operatorname{det} A=-1$ and hence $A \neq I$. Therefore, for any torus bundle over the Klein bottle, $b_{1}$ equals either 2 or 1 . Furthermore, for a Lagrangian torus bundle we have that $b_{1} \neq 1$. Indeed, $\chi=0$ because it is a torus bundle. Therefore, $2-2 b_{1}+b_{2}=0$, but for $M$ to be symplectic we must have $b_{2} \neq 0$.

According to Ue [41, p. 483], by applying bundle isomorphisms one can see that any torus bundle over the Klein bottle having $b_{1}=2$ must be equivalent to one of

$$
M_{i \lambda a}:=\left\{\left(\begin{array}{cc}
-1 & \delta \\
0 & 1
\end{array}\right),\left(\begin{array}{cc}
1 & \lambda \\
0 & 1
\end{array}\right) ;(a, \epsilon)\right\},
$$

where $i=0,1,2,3$ according to whether $(\delta, \epsilon)$ equals $(0,0),(1,0),(0,1)$ or $(1,1)$, respectively. One may assume $\lambda \geq 0, a \geq 0$ and $0 \leq a<\lambda$ if $\lambda>0$.

To verify that these bundles admit symplectic structures with respect to which the fibration is Lagrangian, it remains to construct an integral affine structure on the Klein bottle $K$ with monodromy $\left\{\left(\begin{array}{cc}-1 & \delta \\ 0 & 1\end{array}\right),\left(\begin{array}{ll}1 & \lambda \\ 0 & 1\end{array}\right)\right\}$. (The Euler class can be introduced at will using Luttinger surgery, as for torus bundles over tori; cf. [29].) Since the monodromy matrices of the torus bundle and the integral affine structure are inverse transposes of each other, define $C=A^{-\mathrm{T}}$ and $D=B^{-\mathrm{T}}$.

Mimicking part of Mishachev's work on Lagrangian torus bundles over tori $[\mathbf{2 9}]$, we note that the structure exists if and only if there are vectors $u, v$ such that the integral affine transformations $(C, u)$ and $(D, v)$ generate a properly discontinuous action of $\pi_{1}(K)$ on $\mathbb{R}^{2}$, where $(C, u) \cdot x:=C x+u$ and, similarly, $(D, v) \cdot x:=D x+v$.

In order for $(C, u)$ and $(D, v)$ to generate an action of $\pi_{1}(K)$ we must have

$$
(C, u)(D, v)=(D, v)^{-1}(C, u)
$$

The left-hand side is

$$
(C D, u+C v)
$$

while the right-hand side is

$$
\left(D^{-1},-D^{-1} v\right)(C, u)=\left(D^{-1} C, D^{-1}(u-v)\right) .
$$

The requirement that $C D=D^{-1} C$ imposes no restriction on $\delta$ and $k$. However, equality of the vectors imposes

$$
\left(D^{-1}-I\right) u=\left(C+D^{-1}\right) v,
$$

i.e.,

$$
\left(\begin{array}{ll}
0 & 0 \\
k & 0
\end{array}\right)\left(\begin{array}{l}
u_{1} \\
u_{2}
\end{array}\right)=\left(\begin{array}{cc}
0 & 0 \\
k+\delta & 2
\end{array}\right)\left(\begin{array}{l}
v_{1} \\
v_{2}
\end{array}\right)
$$


Thus the only constraint imposed by the existence of an action is

$$
\lambda u_{1}=(\lambda+\delta) v_{1}+2 v_{2} .
$$

The transformation $(C, u)$ has $x$ as a fixed point if and only if there is a solution $x$ to the equation $(I-C) x=u$. Since

$$
I-C=\left(\begin{array}{cc}
2 & 0 \\
-\delta & 0
\end{array}\right)
$$

we merely need to assure that $\left(u_{1}, u_{2}\right)$ is not proportional to $(2,-\delta)$.

Meanwhile, to prevent $(D, v)$ from having a fixed point we need that $(I-D) x=v$ have no solution. Since

$$
I-D=\left(\begin{array}{ll}
0 & 0 \\
k & 0
\end{array}\right)
$$

it suffices to have $v_{1} \neq 0$.

Choosing $u_{1}=v_{1}=1$, we have an action provided we choose $v_{2}=$ $\frac{1}{2}\left(k u_{1}-(k+\delta) v_{1}\right)=\frac{-\delta}{2}$.

Then we can twist the bundle as described in Section 1 while maintaining the fibers being Lagrangian, i.e., by performing Luttinger surgery $[\mathbf{2 6}]$, to obtain $N(\delta, \lambda ; a, b)$ for any $a, b \in \mathbb{Z}$. Then, as pointed out by Ue $[\mathbf{4 1}$, p. 483], we can change the cross section to assure that, without changing the monodromy matrices, we can arrange that $b \in\{0,1\}$.

6.5.2. Equivalences. There are numerous diffeomorphism equivalences among the $N(\delta, \lambda ; a, \epsilon)$; however, they are so tedious to detail we refer the reader to Theorem 4(ii) in [41] for the exact conditions. However, if one wants to check the equivalence of two particular bundles, it may suffice to note the following two lemmas.

Lemma 6.9 ([41]). The group of bundle isomorphisms of a torus bundle over the Klein bottle $K$ is generated by isomorphisms that take $\left\{A, B ;\left(\begin{array}{l}a \\ b\end{array}\right)\right\}$ to

$$
\begin{aligned}
& \left\{P^{-1} A P, P^{-1} B P ; P^{-1}\left(\begin{array}{l}
a \\
b
\end{array}\right)\right\}, \\
& \left\{A^{-1}, B ; B^{-1} A^{-1}\left(\begin{array}{l}
a \\
b
\end{array}\right)\right\}, \\
& \left\{A, B^{-1} ;-B\left(\begin{array}{l}
a \\
b
\end{array}\right)\right\}, \\
& \left\{B A B^{-1}, B ; B\left(\begin{array}{l}
a \\
b
\end{array}\right)\right\}, \\
& \left\{A^{-1} B, A^{-1} B A ; A^{-2}\left(\begin{array}{l}
a \\
b
\end{array}\right)\right\},
\end{aligned}
$$


and

$$
\left\{A, B ;\left(\begin{array}{l}
a \\
b
\end{array}\right)+B^{-1} A\left(B^{-1}-E\right)\left(\begin{array}{l}
p \\
q
\end{array}\right)+\left(B^{-1} A+I\right)\left(\begin{array}{l}
s \\
t
\end{array}\right)\right\}, p, q, s, t \in \mathbb{Z} .
$$

Lemma 6.10 ([41, pp. 483-484]). The $T^{2}$ bundles over $K$ having $b_{1}=2$ are diffeomorphic and $T^{2}$ bundles over $T^{2}$ as follows:

$$
N\left\{\left(\begin{array}{cc}
-1 & 0 \\
0 & 1
\end{array}\right),\left(\begin{array}{cc}
1 & k \\
0 & 1
\end{array}\right) ; a, 0\right\} \simeq M\left\{\left(\begin{array}{cc}
-1 & -a \\
0 & -1
\end{array}\right),\left(\begin{array}{cc}
1 & k \\
0 & 1
\end{array}\right) ; 0,0\right\}
$$

$$
N\left\{\left(\begin{array}{cc}
-1 & 0 \\
0 & 1
\end{array}\right),\left(\begin{array}{cc}
1 & k \\
0 & 1
\end{array}\right) ; a, 1\right\} \simeq M\left\{\left(\begin{array}{cc}
-1 & 2 a \\
0 & -1
\end{array}\right),\left(\begin{array}{cc}
1 & k \\
0 & 1
\end{array}\right) ; a, 1\right\}
$$

$$
N\left\{\left(\begin{array}{cc}
-1 & 1 \\
0 & 1
\end{array}\right),\left(\begin{array}{cc}
1 & k \\
0 & 1
\end{array}\right) ; a, 0\right\} \simeq M\left\{\left(\begin{array}{cc}
-1 & -a \\
0 & -1
\end{array}\right),\left(\begin{array}{cc}
1 & k \\
0 & 1
\end{array}\right) ; 1,0\right\}
$$

$$
N\left\{\left(\begin{array}{cc}
-1 & 1 \\
0 & 1
\end{array}\right),\left(\begin{array}{cc}
1 & k \\
0 & 1
\end{array}\right) ; a, 1\right\} \simeq M\left\{\left(\begin{array}{cc}
-1 & 2 a+1 \\
0 & -1
\end{array}\right),\left(\begin{array}{cc}
1 & k \\
0 & 1
\end{array}\right) ; a, 1\right\} .
$$

Because the diffeomorphism classification of torus bundles over tori with $b_{1}=2$ agrees with the isomorphism classification, this allows one to check diffeomorphism equivalence of the torus bundles over the Klein bottle. For simplicity, the presentations of the bundles in the above lemmas differ from the presentations in [34] by invoking Theorem 6.3 and Equation (6.50) to change the signs of various entries.

\section{Other classifications}

In this section we briefly discuss various other classifications of almost toric manifolds that one might seek, indicating what is known and what remains to be done to achieve such classifications.

7.1. Fiber-preserving symplectomorphism. The problem of determining the data required to specify a Lagrangian fibration up to fiber-preserving symplectomorphism has been studied in several cases:

(1) Duistermaat [10] solved this problem for regular Lagrangian fibrations of symplectic manifolds of any even dimension: one needs the affine structure of the base and a Lagrangian Chern class that measures the obstruction to the existence of a Lagrangian section. 
(2) In the toric case this was established by Delzant [8] for closed manifolds of any even dimension $2 n$. Here the moment map image modulo equivalence under the action of $\operatorname{Aff}(n, \mathbb{Z})$ is a complete invariant.

(3) Boucetta and Molino [7] solved this problem for locally toric fibrations in any even dimension. The data consist, in our language, of the base $(B, \mathcal{A})$ and a generalization of Duistermaat's Lagrangian Chern class. They also determined that any choice of Lagrangian Chern class and locally toric base $(B, \mathcal{A})$ can be realized by such a fibration.

(4) Zung [45] made a significant generalization to fibrations with a class of singularities he calls "non-degenerate topologically stable." In this case, the data consist of the base $(B, \mathcal{A})$, the fiber-preserving symplectomorphism type of the neighborhood of each singular fiber and an appropriately generalized Lagrangian Chern class. The work of $\mathrm{Vu}$ Ngoc S $[\mathbf{4 3}]$ shows that the structure of the fibration near a singular fiber is delicate information already in dimension four where he found a Fourier series type invariant for the neighborhood of a focus-focus (nodal) singularity.

For closed four-manifolds one could hope for a complete classification that specifies what fibrations can occur. (In higher dimensions one must first surmount the difficulty of even specifying what bases can occur.)

(1) For regular Lagrangian fibrations of four-manifolds over the torus this problem was completely solved by Mishachev [29]. For regular Lagrangian fibrations over the Klein bottle one could follow Mishachev's approach.

(2) For toric fibrations this amounts to Delzant's theorem [8] and the classification of polytopes satisfying the appropriate integrality conditions at each vertex.

(3) To extend to locally toric fibrations in dimension four one needs to treat the cases when the base is a cylinder, Möbius band, torus or Klein bottle. The first two cases amount to an easy exercise since the Lagrangian Chern class vanishes and the possible bases $(B, \mathcal{A})$ are easy to specify. Meanwhile, the second two cases coincide with the classification of regular Lagrangian fibrations.

(4) Extending to the almost toric case would require an understanding of all the integral affine structures with nodes that can occur on $S^{2}$. This question is of independent interest in the context of mirror symmetry (cf. $[19,24]$ ).

7.2. Global symplectomorphism. If one has a classification up to fiber-preserving symplectomorphism this problem amounts to deciding which fibrations are equivalent via a global symplectomorphism. Even in the case of closed toric manifolds this is a non-trivial problem being studied by Yael Karshon, Liat Kessler and Martin Pinsonnault [23]. 
Mishachev [29] conjectured that two Lagrangian torus bundles over a torus are symplectomorphic if and only if they are fiber-preserving symplectomorphic; however, it is not clear how to prove such a conjecture. Meanwhile the special case of the K3 surface is very interesting and unsolved.

7.3. Weak deformation. If two Lagrangian fibrations are known to have diffeomorphic total spaces, one can ask whether the pull back (via some diffeomorphism) of one symplectic structure can be connected to the other symplectic structure via a path of symplectic structures, i.e., whether they are weakly deformation equivalent. One way to verify such a relationship is to connect the two fibrations by a path of fibrations. We conjecture, for instance, that the symplectic structures on any pair of almost toric K3 surfaces are weakly deformation equivalent.

\section{References}

[1] V.I. Arnold, Mathematical methods of classical mechanics, Springer-Verlag, New York, 1978 (Translated from the Russian by K. Vogtmann and A. Weinstein, Graduate Texts in Mathematics, 60).

[2] M. Audin, The topology of torus actions on symplectic manifolds, Birkhäuser Verlag, Basel, 1991.

[3] L.M. Bates, Monodromy in the champagne bottle, Z. Angew. Math. Phys. 42(6) (1991), 837-847.

[4] V.V. Batyrev, Mirror symmetry and toric geometry, in Proceedings of the International Congress of Mathematicians (Berlin, 1998), Vol. II, number Extra Vol. II, (1998) 239-248 (electronic).

[5] J.-P. Benzecri, Notes on a theorem of Benzecri, Notes taken by W. Goldman.

[6] F. Beukers and R. Cushman, The complex geometry of the spherical pendulum, in Celestial mechanics (Evanston, IL, 1999), Contemp. Math. 292. Amer. Math. Soc., Providence, RI, 2002, 47-70.

[7] M. Boucetta and P. Molino, Géométrie globale des systèmes hamiltoniens complètement intégrables: fibrations lagrangiennes singulières et coordonnées actionangle à singularités, C. R. Acad. Sci. Paris Sér. I Math. 308(13) (1989), 421-424.

[8] T. Delzant, Hamiltoniens périodiques et images convexes de l'application moment, Bull. Soc. Math. France 116(3) (1988), 315-339.

[9] S.K. Donaldson, Lefschetz pencils on symplectic manifolds, J. Differential Geom. 53(2) (1999), 205-236.

[10] J.J. Duistermaat, On global action-angle coordinates, Comm. Pure Appl. Math. 33(6) (1980), 687-706.

[11] Y. Eliashberg, Filling by holomorphic discs and its applications, in Geometry of lowdimensional manifolds, 2 (Durham, 1989), London Math. Soc. Lecture Note Ser. 151. Cambridge Univ. Press, Cambridge, 1990, 45-67.

[12] W. Fulton, Introduction to toric varieties, The William H. Roever Lectures in Geometry, Princeton University Press, Princeton, NJ, 1993.

[13] H. Geiges, Symplectic structures on $T^{2}$-bundles over $T^{2}$, Duke Math. J. 67(3) (1992), $539-555$. 
[14] R.E. Gompf, The topology of symplectic manifolds, Turkish J. Math. 25(1) (2001), 43-59.

[15] R.E. Gompf and A.I. Stipsicz, 4-Manifolds and Kirby calculus, American Mathematical Society, Providence, RI, 1999.

[16] P. Griffiths and J. Harris, Principles of algebraic geometry, Pure and Applied Mathematics, Wiley-Interscience [John Wiley \& Sons], New York, 1978.

[17] M. Gromov, Pseudoholomorphic curves in symplectic manifolds, Invent. Math. 82(2) (1985), 307-347.

[18] M. Gross and B. Siebert, Affine manifolds, log structures, and mirror symmetry, Turkish J. Math. 27(1) (2003), 33-60, arXiv:math.AG/0211094.

[19] M. Gross and B. Siebert, Mirror symmetry via logarithmic degeneration data. I, J. Differential Geom. 72(2) (2006), 169-338, arXiv:math.AG/0309070.

[20] M. Gross and P.M.H. Wilson, Large complex structure limits of K3 surfaces, J. Differential Geom. 55(3) (2000), 475-546.

[21] A. Hatcher, Basic topology of 3-manifolds, available at www.math.cornell. edu/hatcher/.

[22] N. Hitchin, Hyper-Kähler manifolds, Astérisque, (206): Exp. No. 748, 3 (1992), $137-166$.

[23] Y. Karshon, Private communication.

[24] M. Kontsevich and Y. Soibelman, Affine structures and non-Archimedean analytic spaces, in The unity of mathematics, Progr. Math. 244. Birkhäuser Boston, Boston, MA, 2006, 321-385.

[25] E. Lerman and S. Tolman, Hamiltonian torus actions on symplectic orbifolds and toric varieties, Trans. Amer. Math. Soc. 349(10) (1997), 4201-4230.

[26] K.M. Luttinger, Lagrangian tori in $\mathbf{R}^{4}$, J. Differential Geom. 42(2) (1995), 220-228.

[27] Y. Matsumoto. Diffeomorphism types of elliptic surfaces, Topology 25(4) (1986), $549-563$.

[28] D. McDuff and M. Symington, Associativity properties of the symplectic sum, Math. Res. Lett. 3(5) (1996), 591-608, dg-ga/9602002.

[29] K.N. Mishachev, The classification of Lagrangian bundles over surfaces, Differential Geom. Appl. 6(4) (1996), 301-320.

[30] B. Moishezon, Complex surfaces and connected sums of complex projective planes, Springer-Verlag, Berlin, 1977 (With an appendix by R. Livne, Lecture Notes in Mathematics, 603).

[31] D.R. Morrison, The geometry underlying mirror symmetry, in New trends in algebraic geometry (Warwick, 1996), London Math. Soc. Lecture Note Ser. 264. Cambridge Univ. Press, Cambridge, 1999, 283-310.

[32] P. Orlik and F. Raymond, Actions of the torus on 4-manifolds. I, Trans. Amer. Math. Soc. 152 (1970), 531-559.

[33] A.A. Oshemkov, Fomenko invariants for the main integrable cases of the rigid body motion equations, in Topological classification of integrable systems, Adv. Soviet Math. 6. Amer. Math. Soc., Providence, RI, 1991, 67-146.

[34] K. Sakamoto and S. Fukuhara, Classification of $T^{2}$-bundles over $T^{2}$, Tokyo J. Math. 6(2) (1983), 311-327. 
[35] A. Strominger, S.-T. Yau and E. Zaslow, Mirror symmetry is T-duality, Nuclear Phys. B 479(1-2) (1996), 243-259.

[36] M. Symington, A new symplectic surgery: the 3-fold sum, Topology Appl. 88(1-2) (1998), 27-53. Symplectic, Contact and Low-dimensional Topology (Athens, GA, 1996).

[37] M. Symington, Symplectic rational blowdowns, J. Differential Geom. 50(3) (1998), 505-518, arXiv:math.DG/9802079.

[38] M. Symington, Generalized symplectic rational blowdowns, Algebr. Geom. Topol. 1 (2001), 503-518 (electronic).

[39] M. Symington, Four dimensions from two in symplectic topology, in Proceedings of the 2001 Georgia International Topology Conference, Proceedings of Symposia in Pure Mathematics, 2003, 153-208.

[40] W.P. Thurston, Some simple examples of symplectic manifolds, Proc. Amer. Math. Soc. 55(2) (1976), 467-468.

[41] M. Ue, On the 4-dimensional Seifert fiberings with Euclidean base orbifolds, in A fête of topology, Academic Press, Boston, MA, 1988, 471-523.

[42] J.-C. van der Meer, The Hamiltonian Hopf bifurcation, Springer-Verlag, Berlin, 1985.

[43] S. Vũ Ngọc, On semi-global invariants for focus-focus singularities, Topology 42(2) (2003), 365-380.

[44] N.T. Zung, A note on focus-focus singularities, Differential Geom. Appl. 7(2) (1997), 123-130.

[45] N.T. Zung, Symplectic topology of integrable Hamiltonian systems. II. Topological classification, Compositio Math. 138(2) (2003), 125-156.

Department of Mathematics

Room 214, Lady Shaw Building

The Chinese University of Hong Kong

Shatin, Hong Kong

E-mail address: leung@math.cuhk.edu.hk

DePARTMENT OF MATHEMATiCS

MERCER UNIVERSITY

1400 Coleman Ave.

MACON, GA 31207

E-mail address: Symington_mf@mercer.edu

Received 7/24/2008, accepted 8/21/2009

The second author would like to thank Tian-Jun Li for pointing out an error in an earlier version, John Etnyre and Eugene Lerman for suggesting useful references, Bill Goldman for providing notes on an argument of Benzecri [5] and Ilia Zharkov for comments on a preliminary draft. The first author was supported in part by NSF/DMS-0103355 and a RGC research grant from the Hong Kong Government. The second author was supported in part by NSF/DMS-0204368. 
